\title{
Integration of Transients in Axisymmetrical Cavities for Accelerators - Formulation and Applications to BNL Photocathode Gun
}

\author{
Zohreh Parsa \\ Brookhaven National Laboratory, Upton, NY 11973 \\ Luca Serafini \\ INFN and University of Milan, Milan, Italy
}

April 1992

Physics Department/CAP

Brookhaven National Laboratory

Associated Universities, Inc.

Upton, Long Island, New York 11973

Under Contract No. DE-AC02-76CH00016 with the UNITED STATES DEPARTMENT OF ENERGY 
Integration of Transients in Axisymmetrical Cavities for Accelerators - Formulation and Applications to BNL Photocathode Gun

\author{
Zohreh Parsa \\ Brookhaven National Laboratory \\ Upton, New York 11973, USA \\ Luca Serafini \\ INFN and University of Milan, Milan, Italy
}

\begin{abstract}
This note provides a sketch of the formalism used for the Integration of Transients in Axisymmetrical Cavities for Accelerators, (ITACA). Application to study the BNL Photocathode Gun via the code ITACA is also included.
\end{abstract}




\section{Introduction}

We present an analysis of the BNL, photocathode gun [1-3] and illustrate the formulation (Section II) used for the integration of the transients in axysymmetrical cavities for accelerators (ITACA).

We perform a numerical study of the interactions between high charged e-beam and the accelerating environment, where the effects of the e.m. fields produced by such interactions (wake-fields) are taken into account in the context of a self-consistent model which integrates the wave-equations for monopole axisymmetric fields coupled to the Newton-Lorentz equations for the motion via the electromagnetic particle in cell code ITACA [4]. With a new charge and current assignment algorithm, based on Rician distributions, which minimize the unphysical fluctuations in the driving current for the wave-equations, the effects of the e.m. interaction on the beam dynamics properties (emittances, energy spreads, etc.) are described (in Section III) for the BNL photoelectron gun. Additional information is given in References [6-10].

\section{Overview of Formalism:}

Consider the inhomogenous wave equations in vacuum:

$$
\begin{gathered}
\nabla^{2} \overrightarrow{\mathrm{H}}-\frac{1}{c^{2}} \frac{\partial^{2} \overrightarrow{\mathrm{H}}}{\partial \mathrm{t}^{2}}=-\nabla \times \overrightarrow{\mathrm{J}} \\
\nabla^{2} \overrightarrow{\mathrm{E}}-\frac{1}{c^{2}} \frac{\partial^{2} \overrightarrow{\mathrm{E}}}{\partial \mathrm{t}^{2}}=\nabla\left(\frac{\rho}{\varepsilon_{0}}\right)+\mu_{0} \frac{\partial \overrightarrow{\mathrm{J}}}{\partial \mathrm{t}}
\end{gathered}
$$

assuming that, both the source

$$
\vec{J}=\vec{J}(r, z, \mathfrak{t}), \rho=\rho(r, z, t)
$$

and the Electromagnetic (E.M.) fields 


$$
\vec{E}=\vec{E}(r, z, t), \vec{H}=\vec{H}(r, z, t)
$$

are axisymmetrical (i.e., cylindrical on axis beams and monopole fields), then one can verify that the wave equation $(1,2)$, can be divided into two uncoupled sets: The TM-like set: $\left(\mathrm{H}_{\phi}, \mathrm{E}_{\mathrm{r}}, \mathrm{E}_{\mathrm{z}}\right)$

$$
\left\{\begin{array}{l}
\frac{\partial^{2} \mathrm{H}_{\phi}}{\partial z^{2}}+\frac{1}{r} \frac{\partial}{\partial r}\left(r \frac{\partial \mathrm{H}_{\phi}}{\partial r}\right)-\frac{\mathrm{H}_{\phi}}{\mathrm{r}^{2}}-\frac{1}{\mathrm{c}^{2}} \frac{\partial^{2} \mathrm{H}_{\phi}}{\partial \mathrm{t}^{2}}=\frac{\partial \mathrm{J}_{z}}{\partial \mathrm{r}}-\frac{\partial \mathrm{J}_{\mathrm{r}}}{\partial \mathrm{z}} \\
\frac{\partial^{2} \mathrm{E}_{\mathrm{r}}}{\partial \mathrm{z}^{2}}+\frac{1}{\mathrm{r}} \frac{\partial}{\partial \mathrm{r}}\left(\mathrm{r} \frac{\partial \mathrm{E}_{\mathrm{r}}}{\partial \mathrm{r}}\right)-\frac{\mathrm{E}_{\mathrm{r}}}{\mathrm{r}^{2}}-\frac{1}{\mathrm{c}^{2}} \frac{\partial^{2} \mathrm{E}_{\mathrm{r}}}{\partial \mathrm{t}^{2}}=\frac{1}{\varepsilon_{\mathrm{o}}} \frac{\partial \rho}{\partial \mathrm{r}}+\mu_{\mathrm{o}} \frac{\partial \mathrm{J}_{\mathrm{r}}}{\partial \mathrm{t}} \\
\frac{\partial^{2} \mathrm{E}_{\mathrm{z}}}{\partial \mathrm{z}^{2}}+\frac{1}{\mathrm{r}} \frac{\partial}{\partial \mathrm{r}}\left(\mathrm{r} \frac{\partial \mathrm{E}_{\mathrm{z}}}{\partial \mathrm{r}}\right)-\frac{1}{\mathrm{c}^{2}} \frac{\partial^{2} \mathrm{E}_{\mathrm{z}}}{\partial \mathrm{t}^{2}}=\frac{1}{\varepsilon_{\mathrm{o}}} \frac{\partial \rho}{\partial z}+\mu_{\mathrm{o}} \frac{\partial \mathrm{J}_{\mathrm{z}}}{\partial \mathrm{r}}
\end{array}\right.
$$

and the TE-like set: $\left(E_{\phi}, H_{r}, H_{2}\right)$

$$
\left\{\begin{array}{l}
\frac{\partial^{2} \mathrm{E}_{\phi}}{\partial \mathrm{z}^{2}}+\frac{1}{\mathrm{r}} \frac{\partial}{\partial \mathrm{r}}\left(\mathrm{r} \frac{\partial \mathrm{E}_{\phi}}{\partial \mathrm{r}}\right)-\frac{\mathrm{E}_{\phi}}{\mathrm{r}^{2}}-\frac{1}{\mathrm{c}^{2}} \frac{\partial^{2} \mathrm{E}_{\phi}}{\partial \mathrm{t}^{2}}=\mu_{\mathrm{o}} \frac{\partial \mathrm{J}_{\phi}}{\partial \mathrm{t}} \\
\frac{\partial^{2} \mathrm{H}_{\mathrm{r}}}{\partial \mathrm{z}^{2}}+\frac{\partial^{2} \mathrm{H}_{\mathrm{r}}}{\partial \mathrm{r}^{2}}-\frac{\mathrm{H}_{\mathrm{r}}}{\mathrm{r}^{2}}-\frac{1}{\mathrm{c}^{2}} \frac{\partial^{2} \mathrm{H}_{\mathrm{r}}}{\partial \mathrm{t}^{2}}=\frac{\partial \mathrm{J}_{\phi}}{\partial \mathrm{z}} \\
\frac{\partial^{2} \mathrm{H}_{z}}{\partial \mathrm{z}^{2}}+\frac{\partial^{2} \mathrm{H}_{z}}{\partial \mathrm{r}^{2}}-\frac{1}{\mathrm{c}^{2}} \frac{\partial^{2} \mathrm{H}_{\mathrm{z}}}{\partial \mathrm{t}^{2}}=-\frac{1}{\mathrm{r}} \frac{\partial(\mathrm{rJ} \phi)}{\partial \mathrm{r}}
\end{array}\right.
$$

The general TM field can be expanded as a sum of infinite $\mathrm{TM}_{\text {onp }}$ modes of a resonant cavity; and the general $T E$ field can be expanded as a sum of infinite $T E_{\text {onp }}$ modes of a resonant cavity where, $\mathrm{J}_{\mathrm{r}}$ and $\mathrm{J}_{\mathrm{z}}$ are the driving current for the TM fi, ld and $\mathrm{J}_{\phi}$ is the driving current for the TE field.

Thus a charged particle q moving with a velocity

$$
\vec{v}=v_{r} \vec{e}_{r}+v_{z} \vec{k}
$$

inside: I) a TM field is subject to a force 


$$
\overrightarrow{\mathrm{F}}=\mathrm{F}_{\mathrm{r}} \overrightarrow{\mathrm{e}}_{\mathrm{r}}+\mathrm{F}_{\mathrm{z}} \overrightarrow{\mathrm{k}}
$$

and gives rise to a current

$$
J=J_{r} \vec{e}_{r}+J_{z} \vec{k}
$$

II) inside a TE field, is subject to a force

$$
\vec{F}=F_{\phi} \overrightarrow{e_{\phi}}
$$

and gives rise to a current

$$
\vec{J}=J_{r} \vec{e}_{r}+J_{\phi} \vec{e}_{\phi}+J_{z} \vec{k}
$$

Then for an initial driving current of the type;

$$
\vec{J}=\left(J_{r}(r, z, t), J_{z}(r, z, t)\right)
$$

in presence of a TM-Field, the sub-system of wave equations for the TM Field

$$
\nabla^{2} \overrightarrow{\mathrm{H}}-\frac{1}{c^{2}} \frac{\partial^{2} \overrightarrow{\mathrm{H}}}{\partial \mathrm{t}^{2}}=-\nabla \times \overrightarrow{\mathrm{J}}
$$

plus the Relativistic equations for the charged particles motion in the TM-Field

$$
\frac{d \vec{p}}{d t}=q(\vec{E}+\vec{v} \times \vec{B})
$$

where $\vec{v}=\left(v_{r}, v_{2}\right)$, is a "self-consistent" system of equations.

In this case; the beam-cavity interaction can be completely described, from the point of view of the field propagations, by means of one scalar equation:

$$
L \Phi-\frac{1}{c^{2}} \frac{\partial^{2} \phi}{\partial t^{2}}=r \frac{\partial J_{z}}{\partial r}-r \frac{\partial J_{r}}{\partial z}
$$

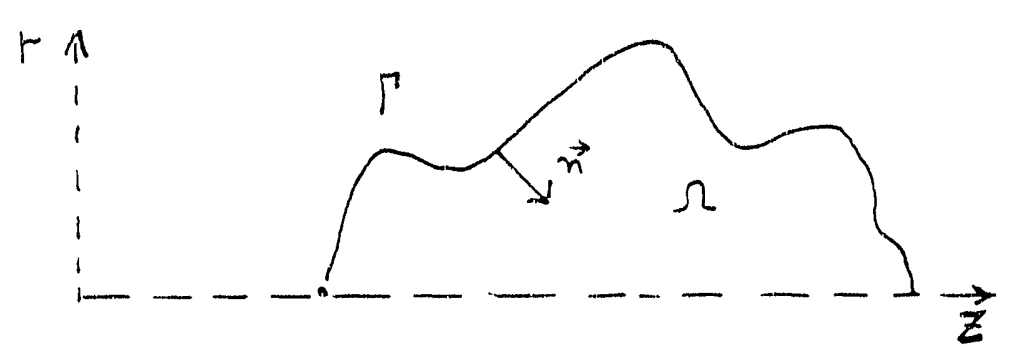

Figure 1. 


$$
\Phi=\mathrm{rH}_{\phi} \text { (pseudo scalar potential) }
$$

where $H_{\phi}=H_{\phi}(r, z, t), J_{r}=J_{r}(r, z, t)$ and $J_{z}=J_{z}(r, z, t)$ and

$$
L \equiv \frac{\partial^{2}}{\partial z^{2}}+\frac{\partial^{2}}{\partial r^{2}}-\frac{1}{r} \frac{\partial}{\partial r}
$$

which is a typical "hyperbolic" equation; with initial conditions $\phi^{0}=\phi(r, z, t=0), \phi^{10}=\frac{\partial \phi}{\partial t}(r, z, t$ $=0), J_{r}^{0}=J_{r}(r, z, t=0)$ and $J_{z}^{0}=J_{z}(r, z, t=0)$, to be solved in a domain $\Omega$, with boundary conditions on the curve $\Gamma$;

$$
\left\{\begin{array}{l}
\left.\frac{\partial \phi}{\partial \eta}\right|_{\Gamma}=0 \\
\phi(r=0)=0
\end{array}\right.
$$

To solve these equations numerically, we adopt the standard FDM (Finite Difference Method) Technique, which allows to transform the differential problem into an algebraic problem, via the discretization of the operator $L$ and of the functions $\phi, J_{r}, J_{z}$ over a (regular) mesh. Thus

$$
\mathrm{L} \Phi \rightarrow \mathrm{L}_{\mathrm{d}} \Phi_{\mathrm{d}}
$$

by expanding the partial derivatives in Taylor series up to the 2 nd order around each point $o(r, z)$ of the mesh.
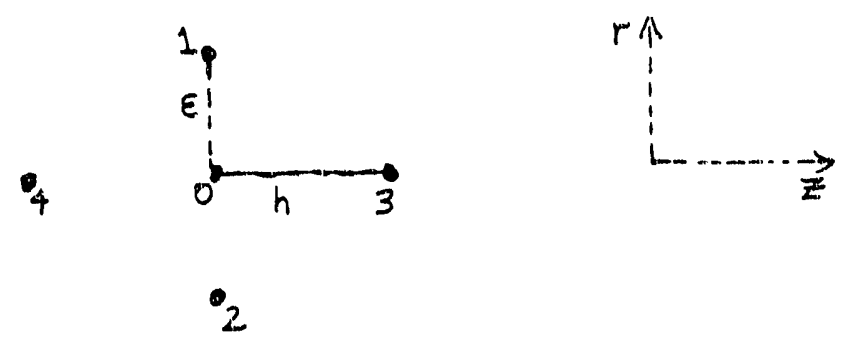

Figure 2. 


$$
\begin{aligned}
\mathrm{L}_{\mathrm{d}} \Phi_{\mathrm{d}}=\mathrm{A} \Phi_{\mathrm{o}}+\mathrm{B}(\mathrm{r}) \Phi_{1}+\mathrm{C}(\mathrm{r}) \Phi_{2}+\mathrm{D} \Phi_{3}+\mathrm{E} \Phi_{4} \\
\mathrm{~A}=\frac{2}{\varepsilon^{2}}+\frac{2}{\mathrm{~h}^{2}} \\
\mathrm{~B}=\frac{1}{\varepsilon^{2}}-\frac{1}{2 \varepsilon \mathrm{r}} \\
\mathrm{C}=\frac{1}{\varepsilon^{2}}+\frac{1}{2 \varepsilon \mathrm{r}} \\
\mathrm{D}=\mathrm{E}=\frac{1}{\mathrm{~h}^{2}}
\end{aligned}
$$

Applying the same procedure to the inhomogeneous term on the r.h.s. of the wave equation (15), we get:

$$
\begin{aligned}
r \frac{\partial J_{z}}{\partial r}-r \frac{\partial J_{r}}{\partial z} & \Rightarrow a(r) J_{z 1}+b(r) J_{z 2}+ \\
& c(v) J_{r 3}+d(r) J_{r 4} \\
\equiv & f_{d}
\end{aligned}
$$

where,

$$
\begin{aligned}
& a=-b=\frac{r}{2 \varepsilon} \\
& c=-d=-\frac{r}{2 h}
\end{aligned}
$$

so that the discretized wave equation becomes:

$$
\left\{\begin{array}{c}
\mathrm{L}_{\mathrm{d}} \Phi_{\mathrm{d}}=-\mathrm{K}^{2} \Phi_{\mathrm{d}} \\
-\mathrm{L}_{\mathrm{d}} \Phi_{\mathrm{d}}+\frac{\partial^{2} \Phi_{\mathrm{d}}}{\partial \tau^{2}}=\mathrm{f}_{\mathrm{d}} \\
\tau=\mathrm{ct}
\end{array}\right.
$$

Since $-L_{d}$ is definite positive one can show that, if $\phi_{d}(r, z, t=0)$ and $f_{d}(r, z, t)$ are sufficiently regular, the explicit time scheme

$$
\frac{\Phi_{d}^{\tau+\delta \tau}-2 \Phi_{d}^{\tau}+\Phi_{d}^{\tau-\delta \tau}}{\delta_{\tau}^{2}}-L_{d} \Phi_{d}^{\tau}=f_{d}^{\tau}
$$

is a 2 nd-order approximation (in $\delta \tau$ ) to the exact solution of the wave-equation. 
This scheme allows to compute directly, for each mesh point $o(r, z)$, the value of $\phi_{0}^{\tau+\delta \tau}$ at the time $\tau+\delta \tau$ for that point, once the value $\phi_{0}^{\tau}$ and $\phi_{0}^{\tau-\delta \tau}$ are known, together with the values at time $\tau$ for all the neighboring points.

If the $\phi$-values of all the mesh points are given at a time $t$ and at $t_{0}-\delta t$, the explicit time scheme, repeatedly applied, allows to compute the field behavior in time, from $t_{0}$ till a given integration time $t_{\max }$. The operations involved are simply matrix multiplications.

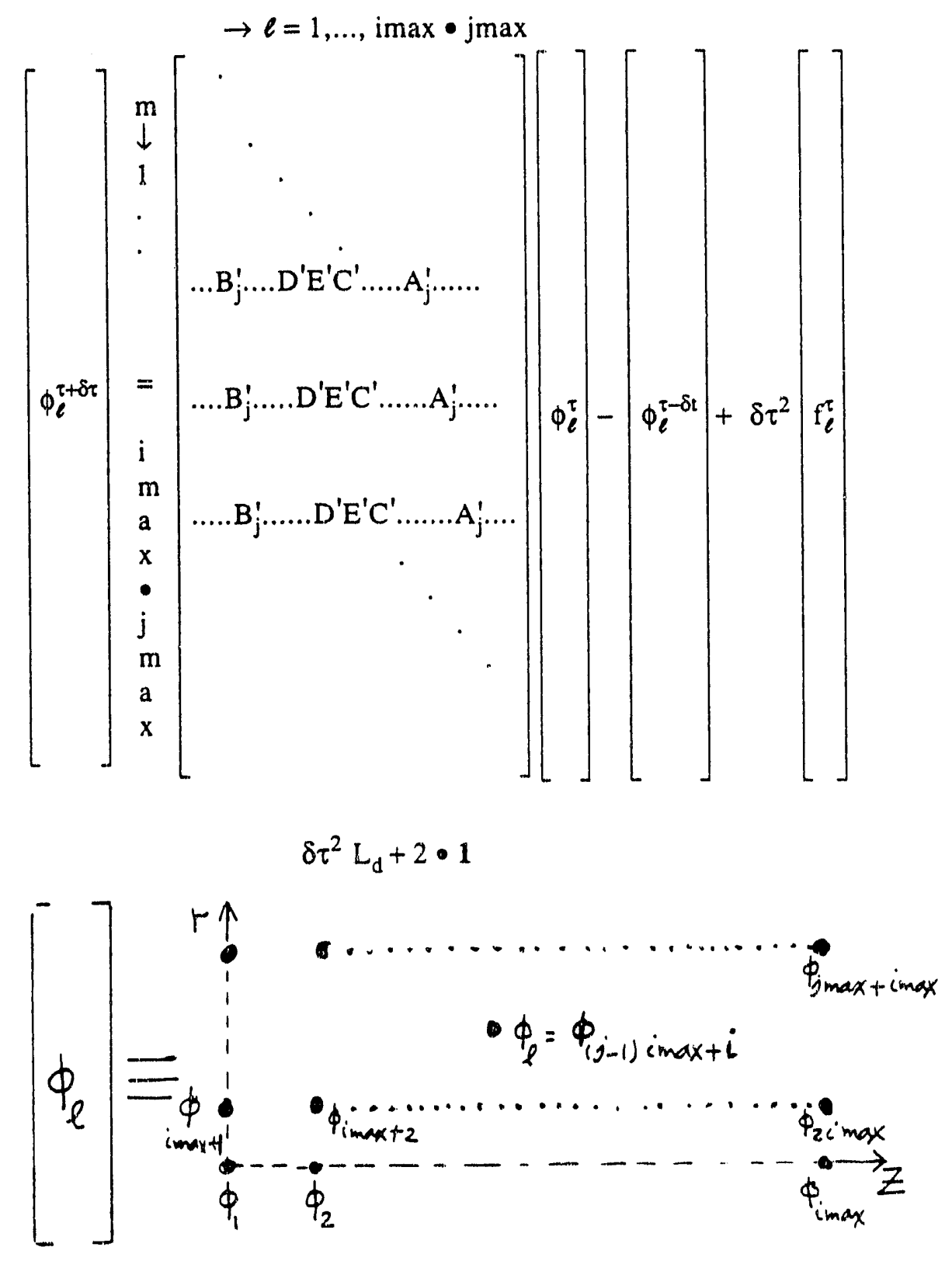


The main disadvantage of a iegular mesh is the difficulty to apply the boundary conditions along curved paths in the $(r, z)$ plane.

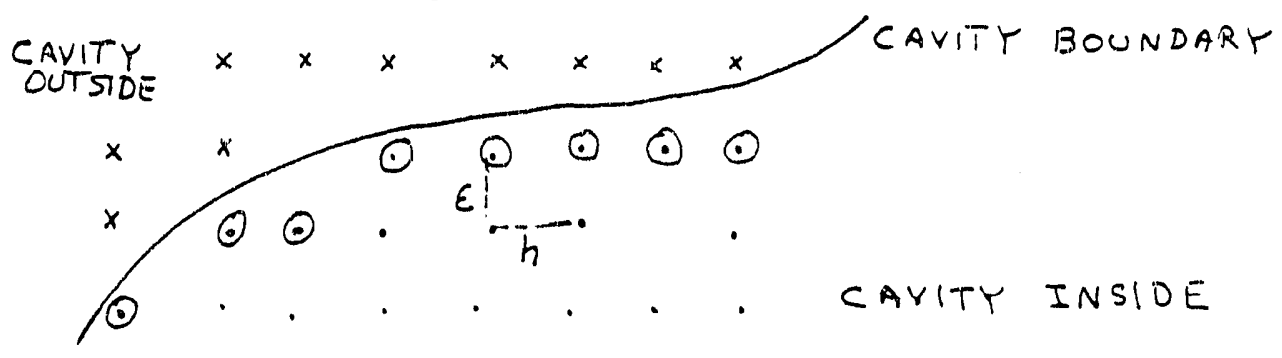

Figure 3.

How is it possible to retain the 2 nd-order approximation in $\varepsilon$ and $h$ also for tl e special internal points $(\mathrm{O})$ lying near to the boundary? (For these points not all the four neighbors live inside the cavity, so the standard scheme is not applicable.)

We expand again up to 2 nd-order in Taylor series the function $L \Phi$ around the point 0 , but we replace the equation for points 1 and 3 , which are actually external points,

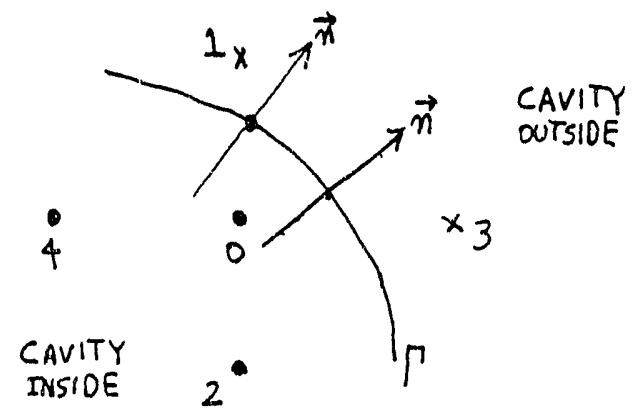

Figure 4.

With the conditions $\frac{\partial \phi}{\partial n}=0$ on the two points $A$ and $B$, which lie on the boundary: the normal direction is specified by the local arc of circle which describes the boundary. 


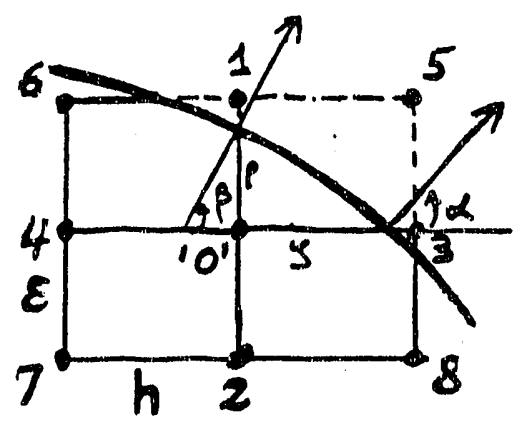

Figure 5.

The wave equation in " 0 " introduces 4 unknowns ${ }^{\tau} \phi_{0},{ }^{\circ} \phi_{r}^{\prime \prime},{ }^{\circ} \phi_{r}^{\prime},{ }^{\circ} \phi_{2}$

$$
\begin{aligned}
& { }^{\circ} \phi_{\mathrm{r}}^{\prime \prime}+{ }^{\circ} \phi_{z}^{\prime \prime}-\frac{1}{r}{ }^{\circ} \phi_{r}^{\prime}=\frac{1}{\tau^{2}}\left[{ }^{\tau} \phi_{0}+{ }^{-\tau} \phi_{0}-2^{\circ} \phi_{0}\right] \\
& \rightarrow\left\{\begin{array}{l}
{ }^{0} \phi_{\mathrm{r}}^{\prime \prime}=\left.\frac{\partial^{2} \phi}{\partial \mathrm{r}^{2}}\right|_{\left({ }^{\prime \prime} \mathrm{o}^{\prime \prime}, \mathrm{t}=\mathrm{t}_{0}\right)} \phi_{\mathrm{z}}^{\prime \prime}=\left.\frac{\partial^{2} \phi}{\partial \mathrm{z}^{2}}\right|_{\left({ }^{\prime \prime} \mathrm{o}^{\prime \prime}, \mathrm{t}=\mathrm{t}_{0}\right)} \\
{ }^{0} \phi_{\mathrm{r}}^{\prime}=\left.\frac{\partial \phi}{\partial \mathrm{r}}\right|_{\left({ }^{\prime \prime} \mathrm{o}^{\prime \prime}, \mathrm{t}=\mathrm{t}_{0}\right)}
\end{array}\right.
\end{aligned}
$$

(For ${ }^{\circ} \phi_{r}^{\prime \prime}$ and ${ }^{\circ} \phi_{z}^{\prime \prime}$; two equations are given at the points 2 and 4 ).

$$
\begin{aligned}
& { }^{\circ} \phi_{2}={ }^{\circ} \phi_{0}-\varepsilon^{0} \phi_{r}^{\prime}+\frac{\varepsilon^{2}}{2}{ }^{o} \phi_{r}^{\prime \prime}+\ldots . . \\
& { }^{\circ} \phi_{4}={ }^{\circ} \phi_{0}-h^{0} \phi_{z}^{\prime}+\frac{h^{2}}{2}{ }^{o} \phi_{z}{ }^{\prime \prime}+\ldots . .
\end{aligned}
$$

which introduces another unknown ${ }^{\circ} \phi_{z}^{\prime}$. For ${ }^{\circ} \phi_{r}^{\prime}$ and ${ }^{\circ} \phi_{z}^{\prime}$; writing $\frac{\partial \phi}{\partial \mathrm{n}}=0$ on the basis of the 2nd-order expansion in $\mathrm{A}$ and $\mathrm{B}$ we obtain equation (25-d and $\mathrm{e}$ ):

(For point 3:)

$$
\begin{aligned}
& \mathrm{n}_{\alpha}=\cos \alpha \hat{\mathrm{k}}+\sin \alpha \hat{\mathrm{e}}_{\mathrm{r}} \Rightarrow \\
& \quad\left({ }^{\circ} \phi_{\mathrm{r}}^{\prime}+\zeta^{\circ} \phi_{\mathrm{rz}}^{\prime \prime}\right) \tan \alpha+{ }^{\circ} \phi_{\mathrm{z}}^{\prime}+\zeta^{\circ} \phi_{\mathrm{z}}^{\prime \prime}=0
\end{aligned}
$$

and (for point 1:) 


$$
\begin{aligned}
& \mathrm{n}_{\beta}=\cos \beta \hat{\mathrm{k}}+\sin \beta \hat{\mathrm{e}}_{\mathrm{r}} \Rightarrow \\
& \quad\left({ }^{\circ} \phi_{\mathrm{r}}^{\prime}+\rho^{0} \phi_{\mathrm{rz}}^{\prime \prime}\right) \tan \beta+{ }^{\circ} \phi_{\mathrm{z}}^{\prime}+\rho^{\circ} \phi_{\mathrm{rz}}^{\prime \prime}=0
\end{aligned}
$$

These two equations introduce another new unknown ${ }^{\circ} \phi_{\mathrm{rz}}$ : Thus to derive another equation we write the expansion for the point 7 :

$$
{ }^{\circ} \phi_{7}={ }^{\circ} \phi_{0}-\varepsilon^{0} \phi_{r}^{\prime}+\frac{\varepsilon^{2}}{2}{ }^{0} \phi_{r}^{\prime \prime}-h^{0}{ }^{0} \phi_{z}^{\prime}+\frac{h^{2}}{2}{ }^{0} \phi_{z}^{\prime \prime}+\varepsilon h^{0} \phi_{r z}^{\prime \prime}
$$

now, solving 6 equations (25a-f), one obtains the following solution for ${ }^{\tau} \phi_{0}$ :

$$
{ }^{+\tau} \phi_{0}=A^{o} \phi_{1}+B^{o} \phi_{2}+C^{\circ} \phi_{3}+D^{\circ} \phi_{4}+E^{o} \phi_{5}+F^{\circ} \phi_{6}+G^{\circ} \phi_{7}+H^{\circ} \phi_{8}+I^{\circ} \phi_{0}+L^{-\tau} \phi_{0}
$$

where

$$
\begin{gathered}
\mathrm{A}=\left(\frac{1+\eta}{2}\right)\left\{2 \frac{\tau^{2}}{\varepsilon^{2}}\left\{1+\frac{2 \rho \tan \beta}{\delta \mathrm{h}}\left[1-\frac{\chi}{\mathrm{D}^{+}}\left(\xi-\frac{\delta \mathrm{h}}{2}\right)\right]\right\}-\frac{\tau^{2}}{\varepsilon \mathrm{h}}\left\{\frac{2 \rho \eta}{\mathrm{h}}\left[1-\frac{\chi}{\mathrm{D}^{+}}\left(\xi-\frac{\delta \mathrm{h}}{2}\right)\right]-\frac{\xi \tan \alpha}{\delta \eta} \frac{\chi}{\mathrm{D}^{+}}\right]\right\} \\
\mathrm{B}=\left(\frac{1-\eta}{2}\right)\left\{2 \frac{\tau^{2}}{\varepsilon^{2}}\left\{1+\frac{2 \rho \tan \beta}{\delta \mathrm{h}}\left[1-\frac{\chi}{\mathrm{D}^{+}}\left(\xi-\frac{\delta \mathrm{h}}{2}\right)\right]\right\}-\frac{\tau^{2}}{\varepsilon \mathrm{h}}\left\{\frac{2 \rho \eta}{\mathrm{h}}\left[1-\frac{\chi}{\mathrm{D}^{+}}\left(\xi-\frac{\delta \mathrm{h}}{2}\right)\right]-\frac{\xi \tan \alpha}{\delta \eta} \frac{\chi}{\mathrm{D}^{+}}\right]\right\} \\
\mathrm{C}=\left(\frac{1+\delta}{2}\right)\left\{2\left(1-\frac{\rho \eta}{\varepsilon}\right)\left[1-\frac{\chi}{\mathrm{D}^{+}}\left(\xi-\frac{\delta \mathrm{h}}{2}\right)\right]-\frac{\chi}{\mathrm{D}^{+}} \delta \mathrm{h}\left(1-\frac{\xi \tan \alpha}{\varepsilon \eta}\right)\right\} \frac{\tau^{2}}{\mathrm{~h}^{2}} \\
\mathrm{D}=\left(\frac{1-\delta}{2}\right)\left\{2\left(1-\frac{\rho \eta}{\varepsilon}\right)\left[1-\frac{\chi}{\mathrm{D}^{+}}\left(\xi-\frac{\delta \mathrm{h}}{2}\right)\right]-\frac{\chi}{\mathrm{D}^{+}} \delta \mathrm{h}\left(1-\frac{\xi \tan \alpha}{\varepsilon \eta}\right)\right\} \frac{\tau^{2}}{\mathrm{~h}^{2}} \\
\mathrm{E}=\left(\frac{1+\eta}{2}\right)\left(\frac{1+\delta}{2}\right) \cdot \frac{\tau^{2}}{\varepsilon \mathrm{h}}\left\{\frac{2 \rho \eta}{\mathrm{h}}\left[1-\frac{\chi}{\mathrm{D}^{+}}\left(\xi-\frac{\delta \mathrm{h}}{2}\right)\right]-\frac{\xi \tan \alpha}{\delta \eta} \frac{\chi}{\mathrm{D}^{+}}\right\} \\
\mathrm{F}=\left(\frac{1+\eta}{2}\right)\left(\frac{1-\delta}{2}\right) \cdot \frac{\tau^{2}}{\varepsilon \mathrm{h}}\left\{\frac{2 \rho \eta}{\mathrm{h}}\left[1-\frac{\chi}{\mathrm{D}^{+}}\left(\xi-\frac{\delta \mathrm{h}}{2}\right)\right]-\frac{\xi \tan \alpha}{\delta \eta} \frac{\chi}{\mathrm{D}^{+}}\right\} \\
\mathrm{H}=\left(\frac{1-\eta}{2}\right)\left(\frac{1-\delta}{2}\right) \cdot \frac{\tau^{2}}{\varepsilon \mathrm{h}}\left\{\frac{2 \rho \eta}{\mathrm{h}}\left[1-\frac{\chi}{\mathrm{D}^{+}}\left(\xi-\frac{\delta \mathrm{h}}{2}\right)\right]-\frac{\xi \tan \alpha}{\delta \eta} \frac{\chi}{\mathrm{D}^{+}}\right\}
\end{gathered}
$$




$$
\begin{aligned}
I=2-\frac{2 \tau^{2}}{\varepsilon^{2}} & \left\{1+\frac{2 \rho \tan \beta}{\delta \mathrm{h}}\left[1-\frac{\chi}{\mathrm{D}^{+}}\left(\xi-\frac{\delta \mathrm{h}}{2}\right)\right]\right\}-\frac{\tau^{2}}{\mathrm{~h}^{2}} \times \\
& \times\left\{2\left(1-\frac{\rho \eta}{\varepsilon}\right)\left[1-\frac{\chi}{\mathrm{D}^{+}}\left(\xi-\frac{\delta \mathrm{h}}{2}\right)\right]-\frac{\chi}{\mathrm{D}^{+}} \delta \mathrm{h}\left(1-\frac{\xi \tan \alpha}{\varepsilon \eta}\right)\right\}
\end{aligned}
$$

$\mathrm{L}=-1$

$D^{+}=\tan \alpha+\frac{2 \tan \beta}{\delta h}\left(\xi-\frac{\delta h}{2}\right)\left(1-\frac{2 \rho \eta}{\varepsilon}\right)$

$\chi=\frac{2 \tan \beta}{\delta h}\left(1-\frac{2 \rho \eta}{\varepsilon}\right)-\frac{2 \eta}{\varepsilon}-\frac{1}{r}$

and

$$
\begin{aligned}
& \rho>0 \quad \eta=-1 \quad \rho>0 \quad \eta=-1 \\
& \xi>0 \stackrel{\leftrightarrow}{\delta} \delta=-1 \quad \xi<0 \quad \delta=1 \\
& \rho<0 \quad \eta=1 \quad \rho<0 \quad \eta=1 \\
& \xi>0 \quad \leftrightarrow \quad \delta=-1 \quad \xi<0 \quad \delta=1
\end{aligned}
$$

The current and charge assignment algorithm is based on a Rician distribution:

$$
\rho_{i}\left(r, r_{i}, z, z_{i}\right)=\frac{q_{i}}{(2 \pi)^{3 / 2} \sigma_{r}^{2} \sigma_{z}} e^{-\frac{\left(z-z_{i}\right)^{2}}{2 \sigma_{z}^{2}}-\frac{\left(r-r_{i}\right)^{2}}{2 \sigma_{r}^{2}}} e^{-X_{I_{0}}(X)}
$$

where $\mathrm{q}_{\mathrm{i}}, \mathrm{r}_{\mathrm{i}}, \mathrm{z}_{\mathrm{i}}$ are the charge and coordinates of the $\mathrm{i}$-th particle; $\sigma_{\mathrm{r}}, \sigma_{\mathrm{z}}$ are the gaussian width of the distribution and $X \equiv \frac{r r_{i}}{\sigma_{r}^{2}}$.

The corresponding current densities distributions are:

$$
\begin{aligned}
& J_{i z}\left(r, r_{i}, z, z_{i}\right)=\beta_{l_{i i}} c \rho\left(r, r_{i}, z, z_{i}\right) \\
& J_{i r}\left(r, r_{i}, z, z_{i}\right)=\beta_{\perp i} c \rho\left(r, r_{i}, z, z_{i}\right) \frac{I_{1}(X)}{I_{0}(X)}
\end{aligned}
$$

with $\beta_{\perp \mathrm{i}}$ and $\beta_{\| \mathrm{i}}$ are the radial and axial components of the $\mathrm{i}$-th particle beta respectively. 


$$
\begin{aligned}
& I_{0}(X)=\frac{1}{\pi} \int_{0}^{\pi} e^{X \cos \theta} d \theta, \\
& I_{1}(X)=\frac{1}{\pi} \int_{0}^{\pi} e^{X \cos \theta} \cos \theta d \theta, \ldots
\end{aligned}
$$

where $I_{0}(X)$ and $I_{1}(X)$ are the 0-th order, 1st-order... modified Bessel functions of the first kind.

Thus

$$
\begin{aligned}
& J_{z}=\beta_{\| l}^{i} \tilde{q}_{i} e^{-\left(z-z_{i}\right)^{2} / 2 \sigma_{z}^{2}} e^{\left(-r^{2}-r_{i}^{2}\right) / 2 \sigma_{r}^{2}} I_{0}\left(\frac{r r_{i}}{\sigma_{r}^{2}}\right) \\
& J_{r}=\beta_{L}^{i} \tilde{q}_{i} e^{-\left(z-z_{i}\right)^{2} / 2 \sigma_{z}^{2}} e^{\left(-r^{2}-r_{i}^{2}\right) / 2 \sigma_{r}^{2}} I_{1}\left(\frac{r r_{i}}{\sigma_{r}^{2}}\right) \\
& \tilde{q}_{i}=\frac{q_{i} c}{(2 \pi)^{3 / 2} \sigma_{r}^{2} \sigma_{z}^{2}}
\end{aligned}
$$

These satisfy the continuity equation, guarantees gauge invariance $(\nabla \cdot \vec{E}-\rho / \varepsilon=0)$ and minimizes the unphysical fluctuation in the driving current. Figure 6 illustrates plot of $\mathrm{J}_{\mathrm{r}}$ vs $\left(r / \sigma_{r}\right)$ for different values of $\left(\frac{r_{i}}{\sigma_{r}}\right)$.

Once the field $\Phi(r, z, \mathfrak{l})=r, H_{\phi}(r, z, t)$ is obtained all over the mesh, via the integration of the wave equation, it is straight forward to compute the electric-field components $\varepsilon_{\mathrm{r}}^{\mathrm{i}, \mathrm{j}}(\mathrm{t}), \varepsilon_{\mathrm{z}}^{\mathrm{i}, \mathrm{j}}(\mathrm{t})$, in each mesh point $(i, j)$, as functions of time, integrating the $2 \cdot$ imax $-j$ small one-dimensional equations: 


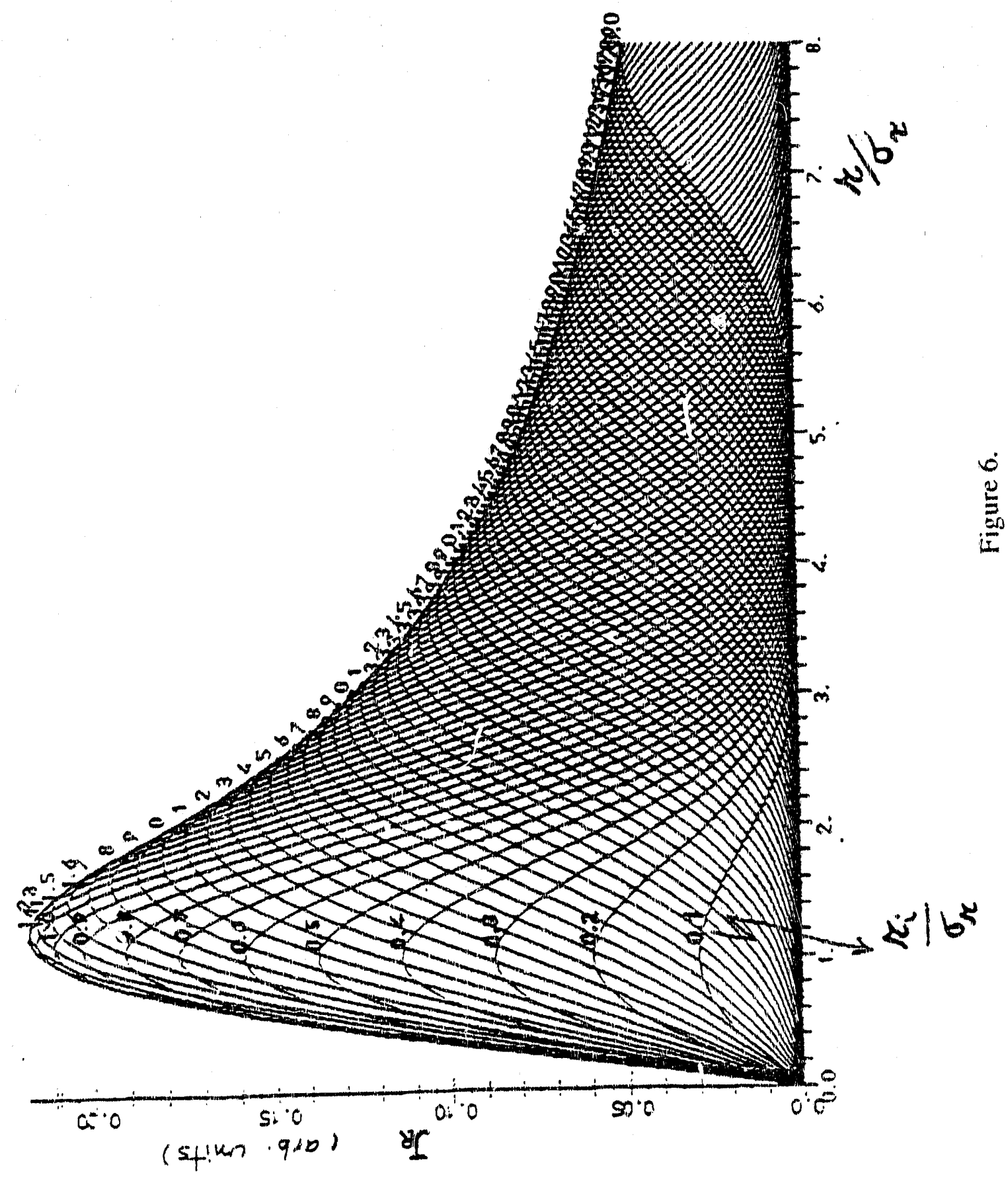




$$
\left\{\begin{array}{l}
\frac{d \varepsilon_{r}^{i, j}}{d \tau}=-\frac{1}{r} \frac{\partial \phi^{i, j}}{\partial z}-J_{i}^{i, j} \\
\frac{d \varepsilon_{z}^{i, j}}{d \tau}=-\frac{1}{r} \frac{\partial \phi^{i, j}}{\partial r}-J_{z}^{i, j} \\
\tau=c t, \vec{\varepsilon}=\frac{\vec{E}}{\sqrt{\mu_{0} / \varepsilon_{0}}} \\
i=1, \ldots ., \text { imax } \\
j=1, \ldots ., \text { jsmall }
\end{array}\right.
$$

where

$$
\varepsilon_{r, z}^{i, j}(t), \phi^{i, j}(t), J_{r, z}^{i, j}(t)
$$

are the values of the electric field, magnetic field and current densities at each point of a subregion of the mesh which covers a region around the $z$-axis where the particles are expected to move.

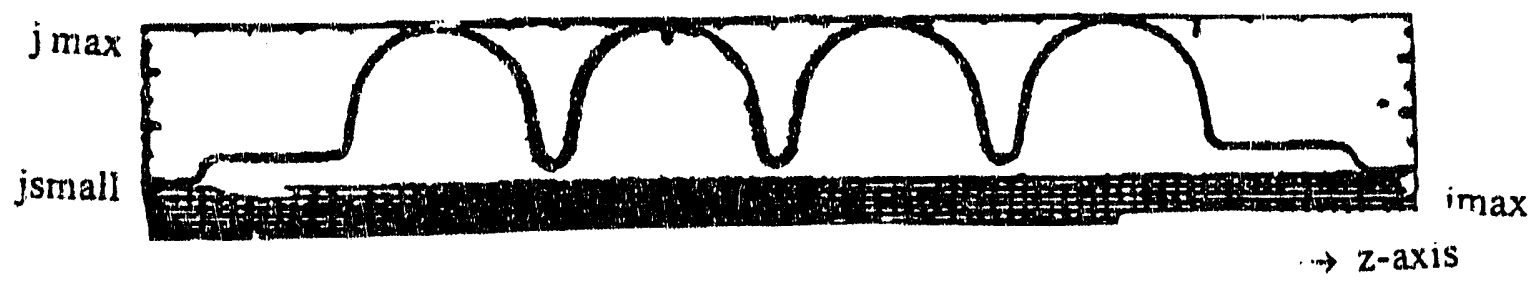

Figure 7.

For large class of particles this saves memory.

For the particle tracking, one must integrate the relativistic equations of the motion, in a TM-like e.m. field: 


$$
\begin{aligned}
\vec{E} & =\left(E_{r}(r, z, t), E_{z}(r, z, t)\right) \\
\vec{H} & =\left(H_{\phi}(r, z, t)\right) \\
\frac{d \beta_{\| n}}{d \tau} & =\frac{\Omega}{\gamma_{n}}\left[\varepsilon_{z}^{n}\left(1-\beta_{\| n}^{2}\right)-\varepsilon_{r}^{n} \beta_{\| n} \beta_{\perp n}+H_{\phi}^{n} \beta_{\perp n}\right] \\
\frac{d \beta_{\perp n}}{d \tau} & =\frac{\Omega}{\gamma_{n}}\left[\varepsilon_{r}^{n}\left(1-\beta_{\perp n}^{2}\right)-\varepsilon_{2}^{n} \beta_{\| n} \beta_{\perp n}-H_{\phi}^{n} \beta_{\| n}\right] \\
\frac{d z_{n}}{d \tau} & =\beta_{\| n} \\
\frac{d r_{n}}{d \tau} & =\beta_{\perp n} \\
\tau & =c t, n=1, \ldots, N, \Omega=\frac{q \mu}{m_{0} c} \\
\gamma_{n} & =\left[1-\beta_{\| n}^{2}-\beta_{\perp n}^{2}\right]^{-1 / 2}
\end{aligned}
$$

where $\varepsilon_{r}^{n}, \varepsilon_{z}^{n}, H_{\phi}^{n}$ are the interpolated values on the mesh at the position $\left(r_{n}, z_{n}\right)$. To integrate these $\mathrm{N}_{\mathrm{p}}$ (number of macro particles) 1 -dim equations, together with the $2 \mathrm{imax} \cdot \mathrm{jsmall}$ equations for the electric field, a standard R.-K. procedure can be used, getting a IV-order approximation.

\section{BNL Photocathode Gun:}

The BNL gun corsists of a $11 / 2$ cell $\pi$-mode cavity with a methal photocathode at the midplane of its first cell. With inner diameter of $83.08[\mathrm{~mm}]$, and total length of $78.75 \mathrm{~mm}$ (where the 1 st cell length $=2.625 \mathrm{~cm}$, and the 2 nd cell length $=5.250 \mathrm{~cm}$, with Radius of aper ture of $1[\mathrm{~cm}]$.

The gun is powered by a (conventional type), SLAC klystron, and is designed to produce a low emittance beam of $4.65 \mathrm{MeV}$ at the exit of the gun, with the $100 \mathrm{MV} / \mathrm{m}$ Field on cathode (i.e., an average axial gradient of $66.6 \mathrm{MV} / \mathrm{m}$ ), cavity shunt impedance of $57 \mathrm{Mohm} / \mathrm{m}$ cavity 
$\mathrm{Q}$ of 11,900 , and the stored energy of -4 Joules [J]. Figure 7 shows the schematic plot of the BNL gun. The initial results are obtained by illuminating the cathode with a Nd: YAG laser beam $(266 \mathrm{~nm})$, where the photoelectrons are injected to the LINAC via a transport beamline.

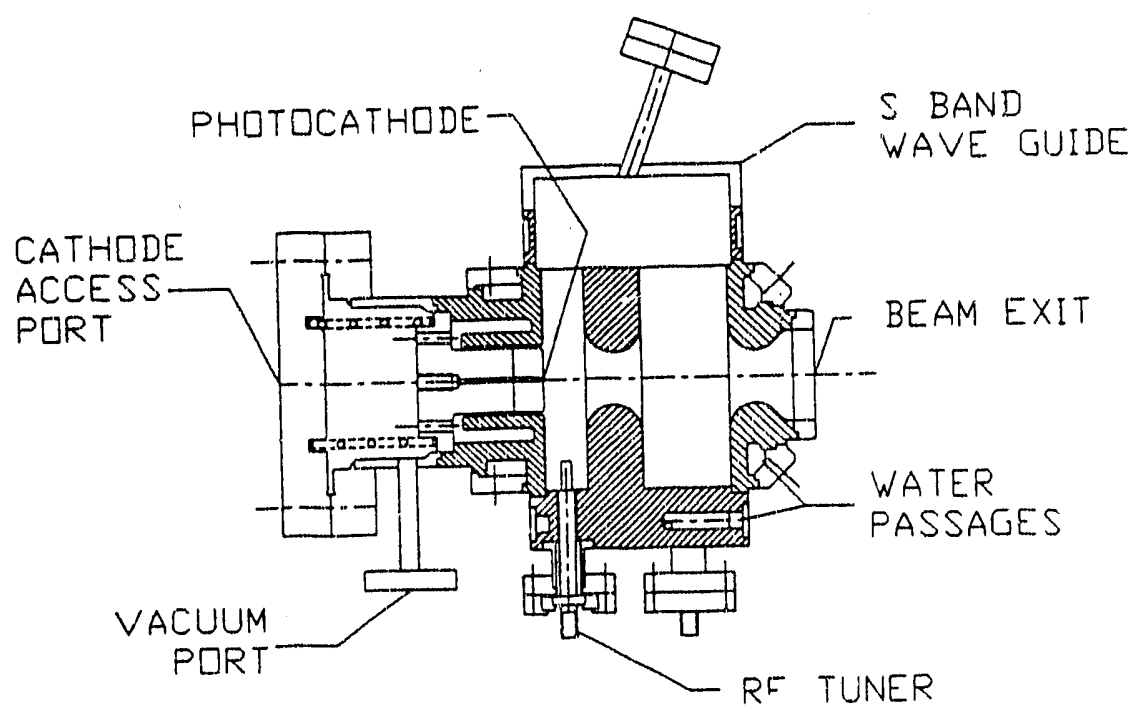

Figure 7. Schematic Plot of the BNL Photocathode Gun.

In the following Figures, I-V, (see Figure Captions below), the behaviour of the beam including the self field and wake field effects (at various points along the cavity) are shown, for the standard BNL gun, where the field on the cathode is $100 \mathrm{MV} / \mathrm{m}$, the laser spot size is 3 $\mathrm{mm}$, the laser pulse width is $2 \mathrm{p}$ second, the initial phase is $63^{\circ}$, and the bunch charge is $1 \mathrm{nc}$.

The injection energy of the photoelectrons is $1.5 \mathrm{eV}$, a typical value for a Yt cathode, with the rms emittance, of $\approx 3.5 \mathrm{~mm}$-mrad at the cathode. The laser-pulse has been taken to have a Gaussian distribution both in the radial coordinate (between $r=0$ and $r=3 \sigma_{r}=9 \mathrm{~mm}$ ) and in the longitudinal direction, where it is described between $-3 \sigma_{t}$ and $3 \sigma_{t}$ from the centroid of the pulse (a total of $12 \mathrm{ps}$ ). 
The initial phase of $63^{\circ}$ (used in this paper) corresponds to the time the head of laser (at $3 \sigma_{t}$ from the centroid) hits the cathode surface. Thus, the injection phase of the centroid of the electron bunch is at $63^{\circ}+3 \sigma_{1}$, which is $\equiv 68^{\circ}$.

In Table A, the variation of the resulis obtained with the change in the injection phase and the cathode material are summarized. For these calculations a mesh with 165,000 points, integration step of $.067 \mathrm{~mm}(\cdot 23 \mathrm{RF}$ deg); and 2400 particles with Gaussian widths of $0.3 \mathrm{~mm}$ radially and $0.15 \mathrm{~mm}$ axially were used for 2 types of cathodes (Yttrium and Cooper). The $\mathrm{ND}-\mathrm{Yag}$ photon energy was assumed to be $4.6 \mathrm{eV}$, the work function of $\mathrm{Cu} 4.6 \mathrm{eV}$ and $\mathrm{Yt}$ to be $3.1 \mathrm{eV}$.

In Table $B$, the variation in the results obtained with changes in mesh size and number of particles are shown (and are negligible).

Figures I-(4 and 5), show the effect of the cavity surface around the cathode onto the bunch self field, at $\left(\phi_{\text {out }} \equiv 83^{\circ}\right)$.

Figures II - $(4,5)$, show the propagation of the bunch self - field, (at $\phi_{\text {out }} \equiv 103^{\circ}$ ).

Figures III - $(4,5)$, show the effect of wake and the bunch self - field, (at $\phi_{\text {out }} \cong 123^{\circ}$ ).

Figures IV-1, shows the self-field plot of the bunch (the magnetic component), and

Figures IV-2, shows the self-field plot of the bunch; (the electric component), at ouput phase of $90 \mathrm{RF}$ deg after the injection (63\%), hence at RF phase of $153^{\circ}$.

In Figure (IV-2), the solid- lines, has a long negative tail produced by the interaction between the bunch charge and current with the cathode surface; (i.e., by the image charge and current).

The peak in the radial electric field (dashed lines) is such that the axial position of the peak is decreasing with increasing radius. That is due to the interaction with the cavity surface in the iris region (i.e., due to wake - field effect: in fact this distribution matches the shape of the field lines in the $\mathrm{r} * \mathrm{H}_{\phi}=$ constant plot of previous graph, Figure (IV-1).

Figure V, shows various parameters with changes in the initial phase values, at $\phi_{\text {out }} \equiv$ $243^{\circ}$. Additional analaysis of the BNL gun is given elsewhere, see e.g., [1,6-10]. 
Table A

Cathode

Starting energy [eV]

Cathode peak field $[\mathrm{MV} / \mathrm{m}]$

Extract bunch charge [ $\mathrm{nC}$ ]

Laser spot $\sigma_{\mathrm{r}}[\mathrm{mm}]$

Rms cathode emitt. [m•rad]

Laser pulse length $\left(2 \sigma_{t}\right)$ [ps]

Injection phase [deg]

Exit charge $[\mathrm{nC}]$

Exit energy [MeV]

Rms energy spread [keV]

Exit radius $\sigma_{\mathrm{x}}[\mathrm{mm}]$

Exit divergence $\sigma_{x_{1}}$ [mrad]

Exit rms nürm. emitt. [m•rad]

Rms bunch length [mm]

Peak current [A]

\section{Copper}

.05

100

1.

3.

$.65 \cdot 10^{-6}$

4.

57.

.89

4.18

$\pm 14$

4.1

5.0

1.15

174

\section{Copper Yttrium Yttrium}

.05

100

1.

3.

$.65 \cdot 10^{-6}$

4.

63.

.89

4.14

$\pm 29.0$

4.1

26.9

7.5

1.20

166
1.5

100

1.

3.

$3.5 \cdot 10^{-6}$

4.

60.

.89

4.17

$\pm 21.6$

4.1

27.8

6.5

1.175

170
1.5

100

1.

3.

$3.5 \cdot 10^{-6}$

4.

63.

.89

4.14 $\pm 29.0$

4.1

26.9

8. 1.20 166

Table B

Mesh

330,000

165,000

6200

2400

Copper

Cathode

Starting energy [eV]

Cathode peak field [mV/m]

.05

Copper

100

Extract. bunch charge $[\mathrm{nC}]$

1.

3.

$.64 \cdot 10^{-6}$

.05

100

1.

3.

$.65 \cdot 10^{-6}$

4.

4.

60.

60.

.89

4.173

.89

4.170

$\pm 21.6$

Exit energy [MeV]

Rms energy spread [keV]

$\pm 21.4$

4.1

Exit radius $\sigma_{x}[\mathrm{~mm}]$

4.1

27.8

Exit divergence $\sigma_{x}$ [mrad]

27.9

5.5

Exit rms norm. emitt. [m・rad]

5.2

$1.17 j$

1.170

170 
Figure VI, shows plot of the transverse emittance vs $z$, for the case of copper cathode at $\Phi_{0}=57^{\circ}$ (first column of Table A).

\section{Conclusion}

We discussed the formalism used for integrating the transients in axisymmetrical cavities (Section II), and illustrated some numerical results obtained via program ITACA for the BNL. photocathode gun (Section III). The preliminary data measured at ATF gave a much smaller value e.g. for emittance as compared to those obtained with ITACA, although for a more accurate comparison additional measurements are needed. They will be made when more data beccormes available. For additional information see references [1-10].

\section{References}

1. Z. Parsa, "Preliminary Emittance Measurements and Calculations of the Laser Excited Beam from the BNL Accelerator Test Facility Radio Frequency Electron Gun", Proceedings of the BNL Center for Accelerator Physics Meetings, Vol. II, Sec. II, P. 1-54, BNL-52292 (1991), and Ref. therein.

2. K. Batchelor, I. Ben-Zvi, I. Biglio, T.S. Chou, R.C. Fernow, J. Fischer, N. Kurnit, K.T. McDonald, R.B. Palmer, Z. Parsa, G. Pellegrini, J. Sheehan, T. Srinivasan-Rao, S. Ulc, A. Van Steenbergen, M. Woodle, "Operational Siatus of Brookhaven Accelerator Test Facility", Proceedings of the 1989 IEEE Particle Accelerator Conf. Chicago, Ill, March 20-23, 1989, \#89CH2669-0 (1990), and BNL Report BNL-41797; ibid., Proceedings of the 2nd European Accelerator Conf. Nice, France, June 12-16, 1990, Ed. Frontiers, (1990).

3. P. Russel et al., The Brookhaven Accelerator Test Facility, Proceeds of the Rice Meeting, 1990 of APS Div. of Particle - Fields, Houston, Tx. Jan. 1990, (P. Bonner and H. Miettinen, eds.) World Scientific, Singapore, P. 975, (1990).

4. L. Serafini and C. Pagani, "A Computer Code for the Integration of Transient Equations in Axisymmetrical Cavities", Proc. 1st. EPAC, Rome, Ed World Scientific, p. 866, (1988); L. 
Serafini et al., "Design of the SC Laser Driven Injector for ARES", Proc. 2nd EPAC Nile, Ed. Frontieres, p. 143 (1990), and Numerical Simulations for the ARES SC Injectors, Tep. INFN TC-90/10.

5. M. Zanin, Thesis, University of Milan (1989); A. Pretti, L. Serafini and M. Zanin (to be published).

6. Z. Parsa, and L. Young, "On Design and Analysis of High Brightness Electron Source", Proceedings, of the 2nd European Particle Accelerator Conf., Nice - France, June 12-16, 1990, Nile, Ed., Frontieres, (1990); BNL Report BNL-44365 (1990).

7. Z. Parsa, and L. Young, The BNL Photocathode Gun with the Code PARMELA", Proceedings of the 13th Int. FEL Conf., Santa Fe, NM., 260-30 Aug., 1991, BNL-46804 (1991).

8. Z. Parsa, "Analysis of a High-Brightness Photoelectron Beam with Self-Field and Wake-Field Effects", ibid and BNL-46794 (1991).

9. Z. Parsa, "Effects of Variation of the Laser Pulse Width on the Laser Induced Photo-electrons", ibid, and BNL-46795 (1991).

10. Z. Parsa, "Interactions Between Charged Paricles and RF Space Charge and Wake Fields in an Accelerating Structure", Proceedings of the Particles and Fieids 91 conf. Aug. 18-22, 1991, Vancouver, Canada, (1991).

* An executable version of the program will be available on BNLDAG in [PARSA.ZPACC].

**We (Z.P.) thank Paul Kessier, Tony Natoli and R. Imossi for providing extra disks and computer assistance, and A. Pretti for assisting with the Graphic Processor. 
Figure I-1 to 8 , at $\phi_{\text {out }}=82.953^{\circ}, \phi_{\text {init }}=63^{\circ}$

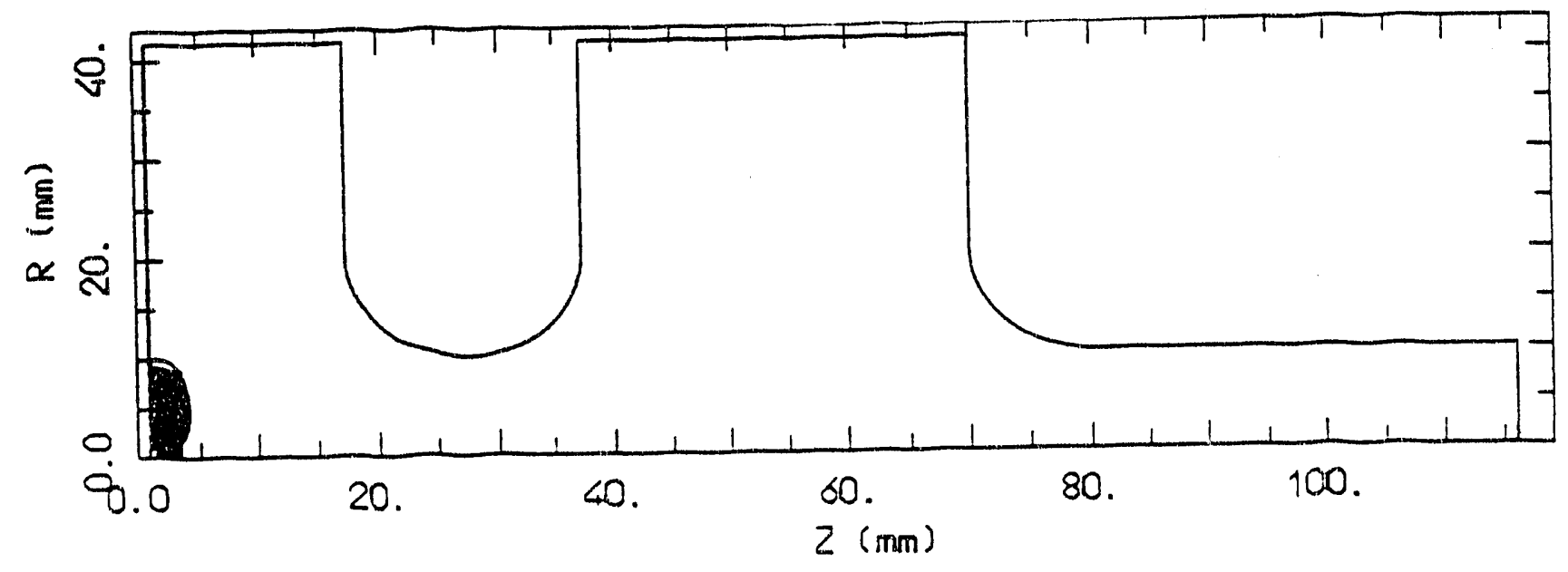

Figure I-1 shows plot of the $\mathrm{r} * \mathrm{H}_{\phi}=$ const lines for $1 \mathrm{nC}$ case, total field. 


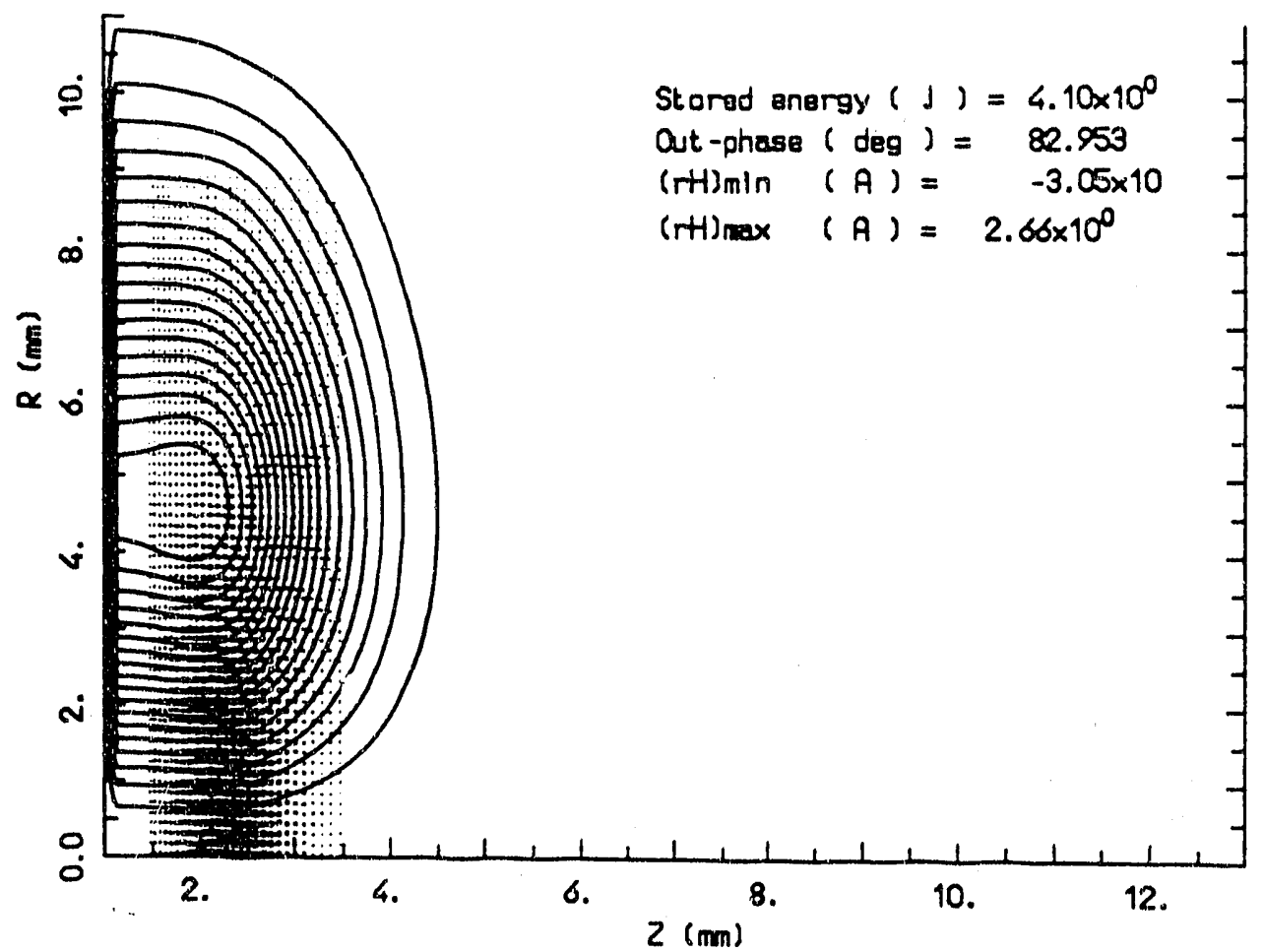

Figure I- 2 shows plot of the lines of self field produced by $1 \mathrm{nC}$ bunch.

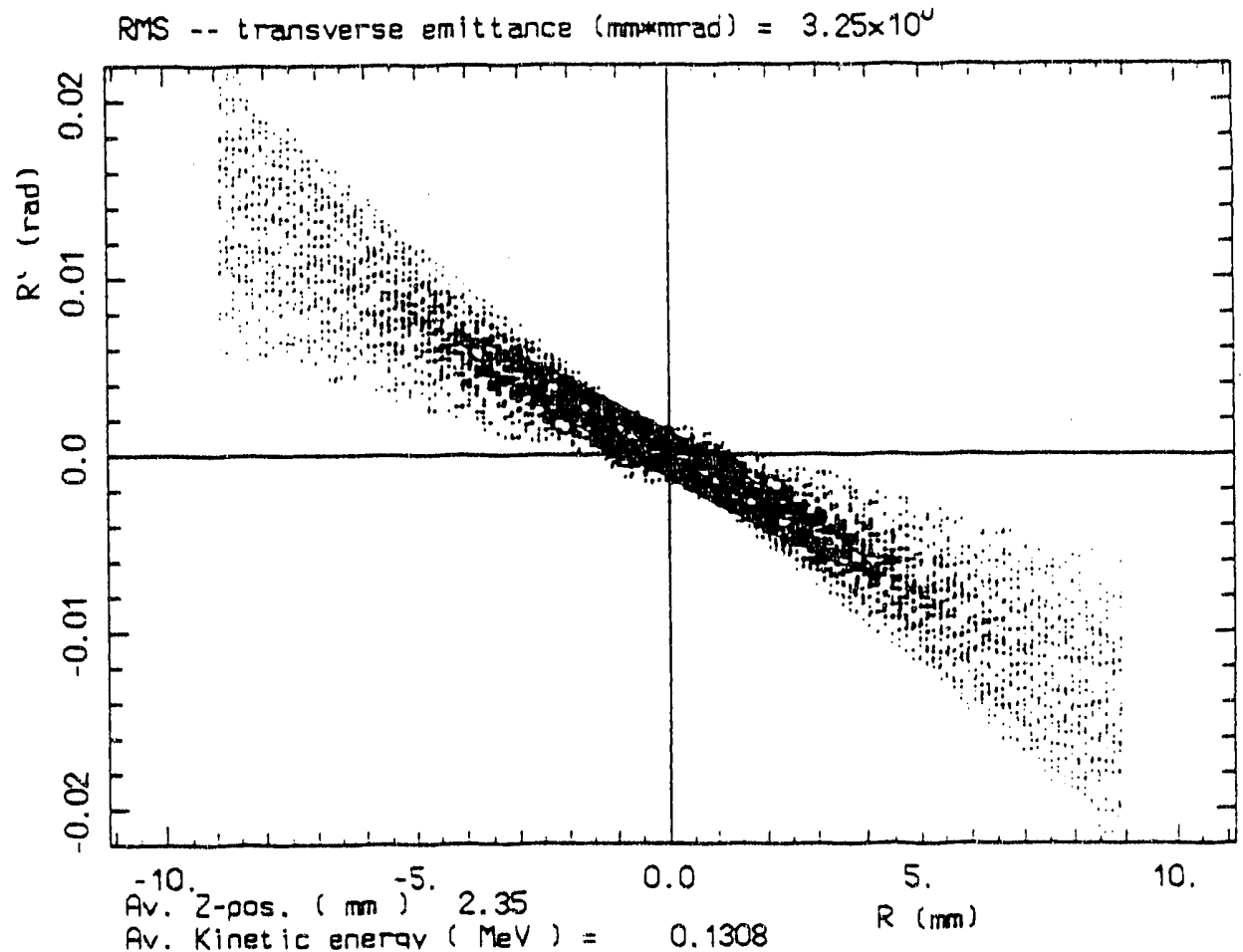

Figure I-3 shows plot of R vs R'; the transverse phase space, 


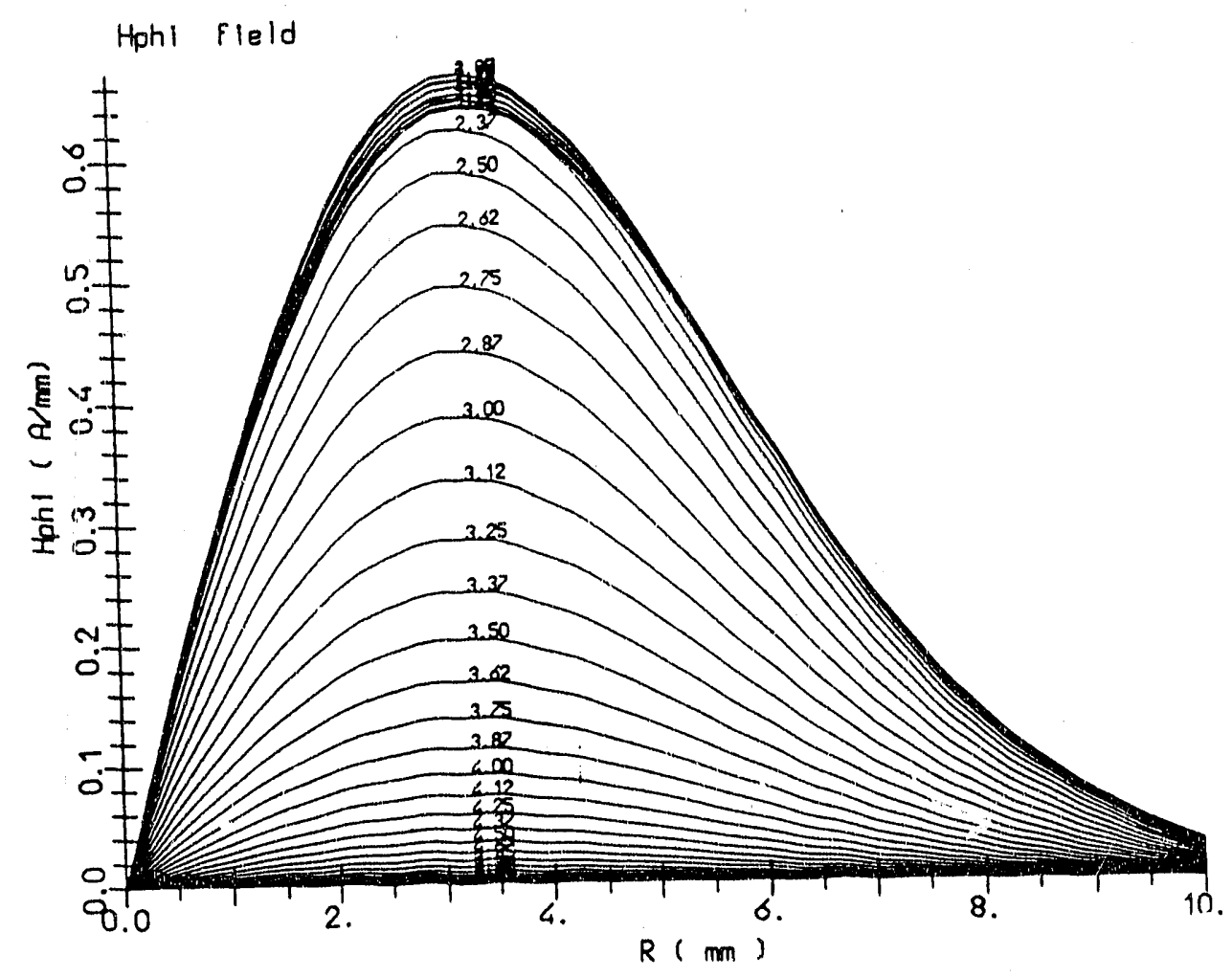

Figure I-4 shows plot of $\mathrm{H}_{\phi}$ vs $\mathrm{R}$,

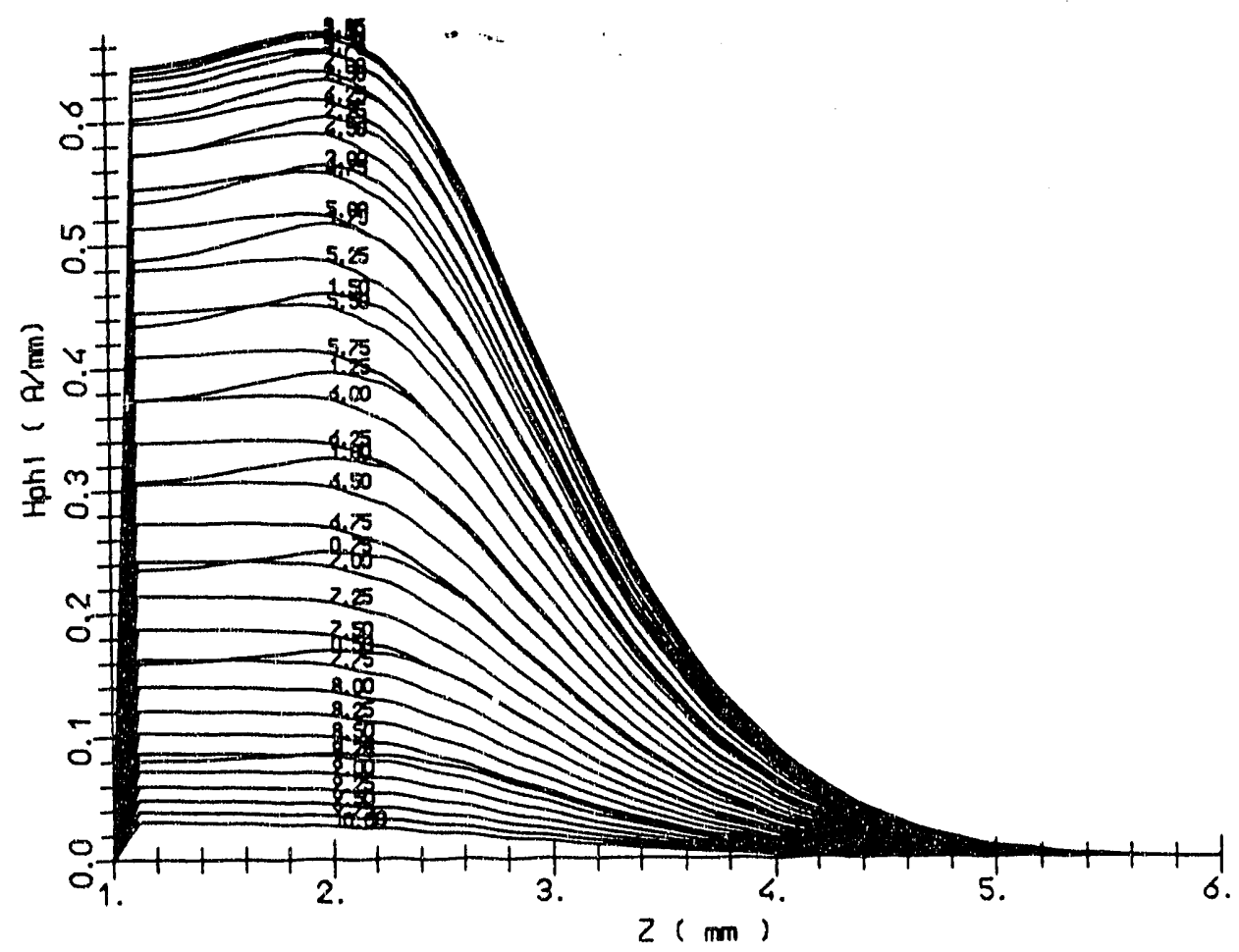

Figure I-5 shows plot of $\mathrm{H}_{\phi}$ vs $\mathrm{z}$, the effect of cavity surface around the cathode onto the bunch self field. 


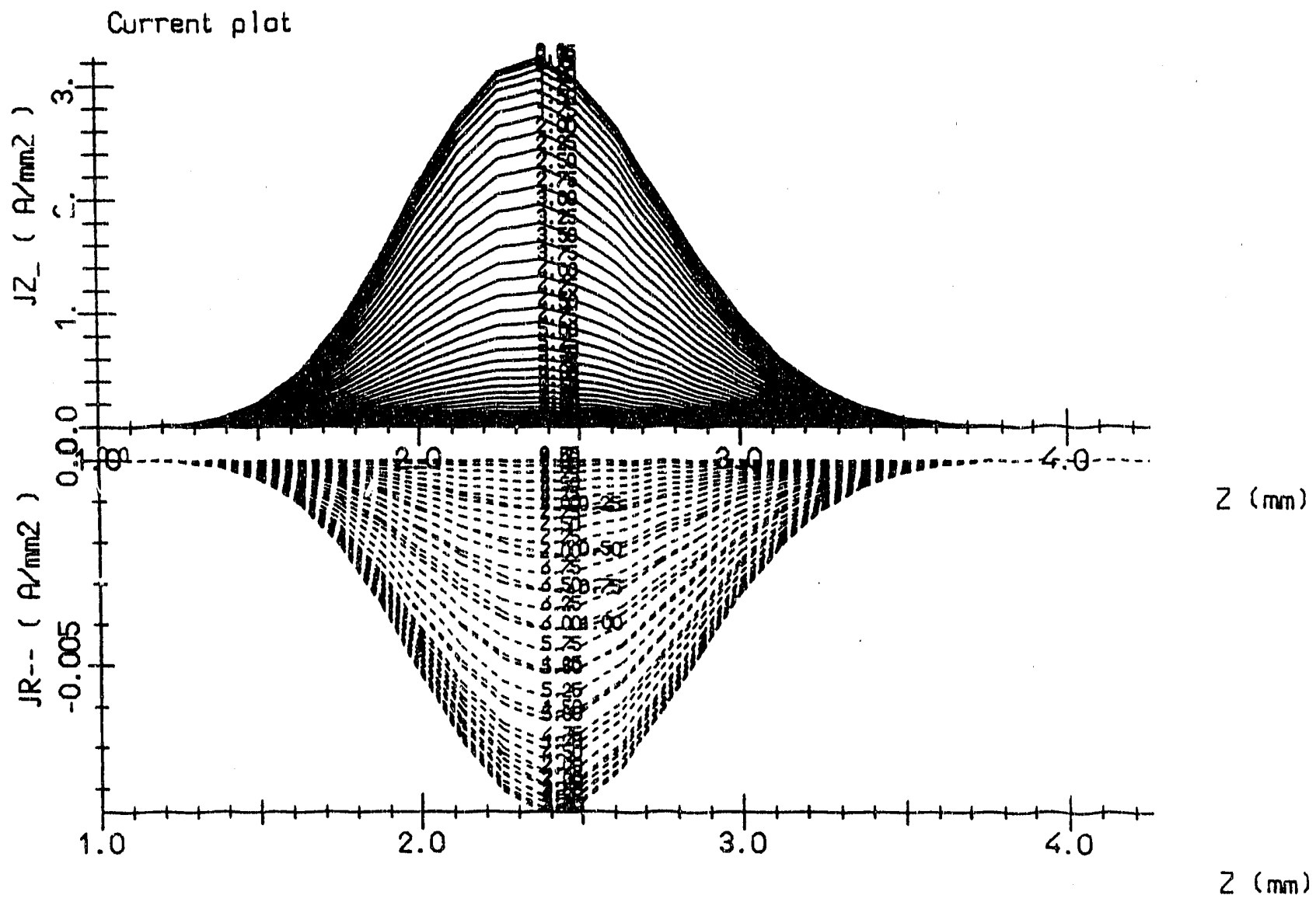

Figure I- 6 shows plot of $\mathrm{Jr}$ (solid lines) and $\mathrm{Jz}$ (dashed lines) distributions as functions of $\mathrm{z}$ at different radii. Note the absence of unphysical fluctuations. 


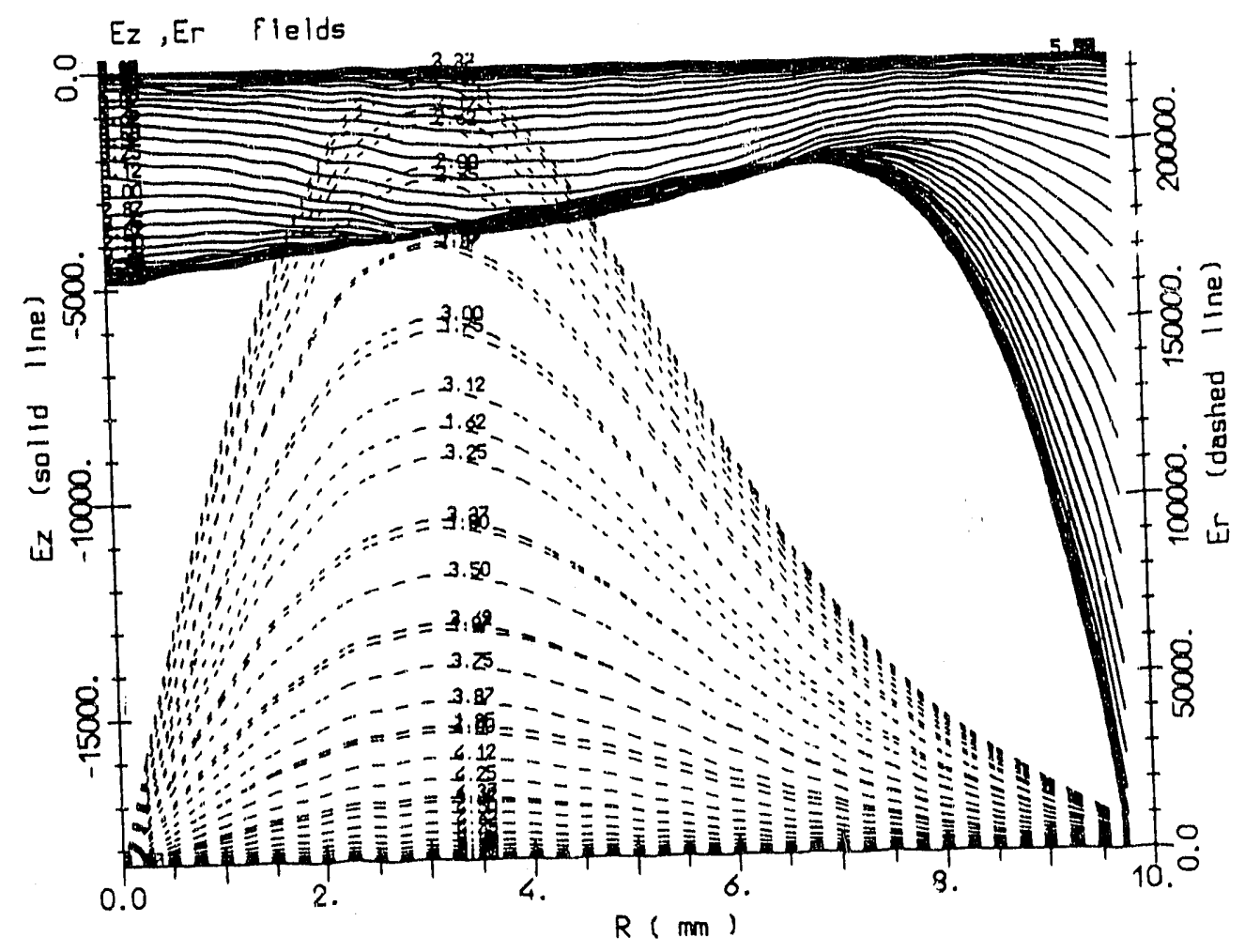

Figure I-7 shows plot of Ez (solid line) and Er (dashed lines) vs $\mathrm{r}$.

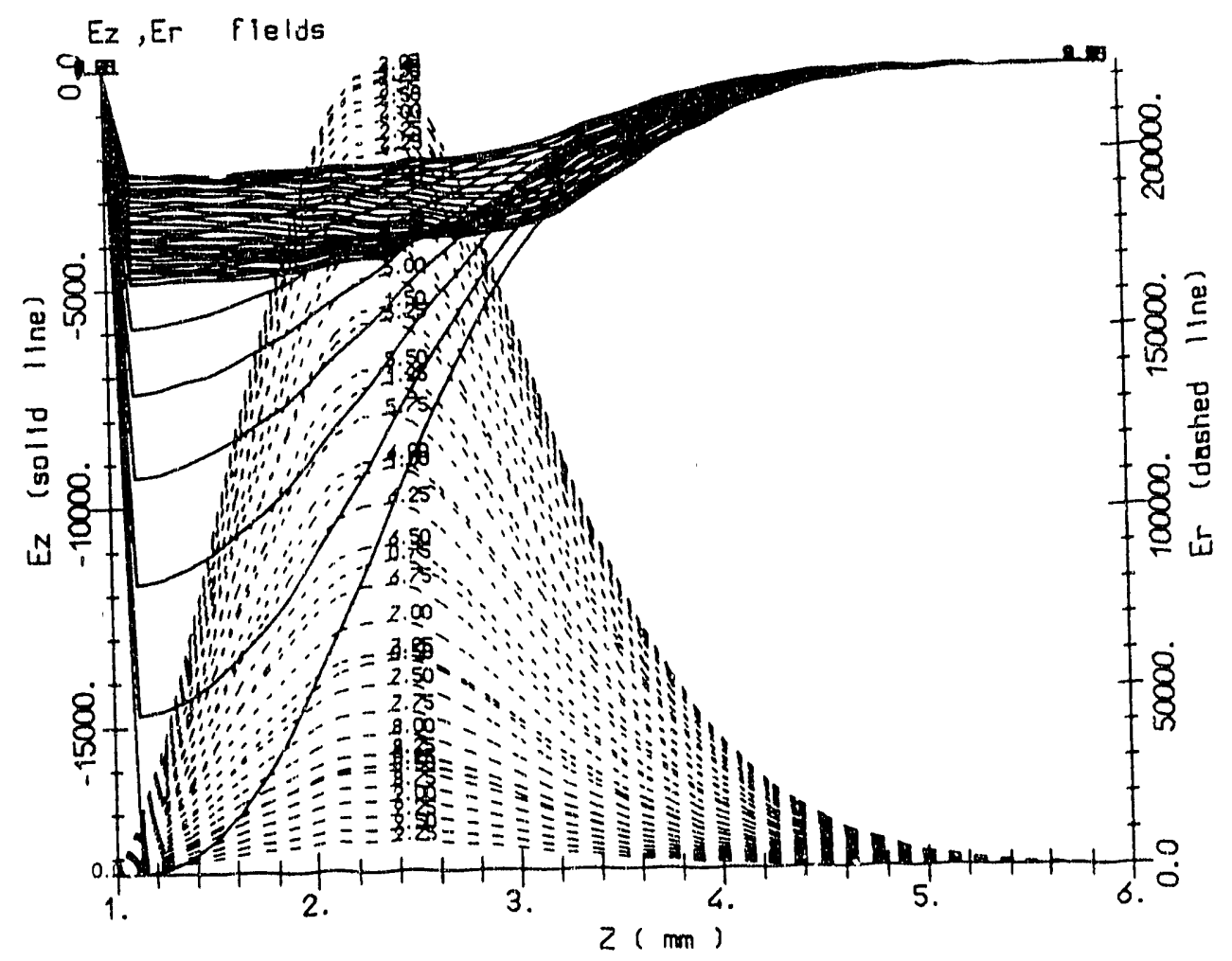

Figure I- 8 shows plot of Ez (solid line) and Er (dashed lines) vs Z. 
Figure II-1 to 8 , at $\phi_{\text {out }}=103^{\circ}, \phi_{\text {init }}=63^{\circ}$.

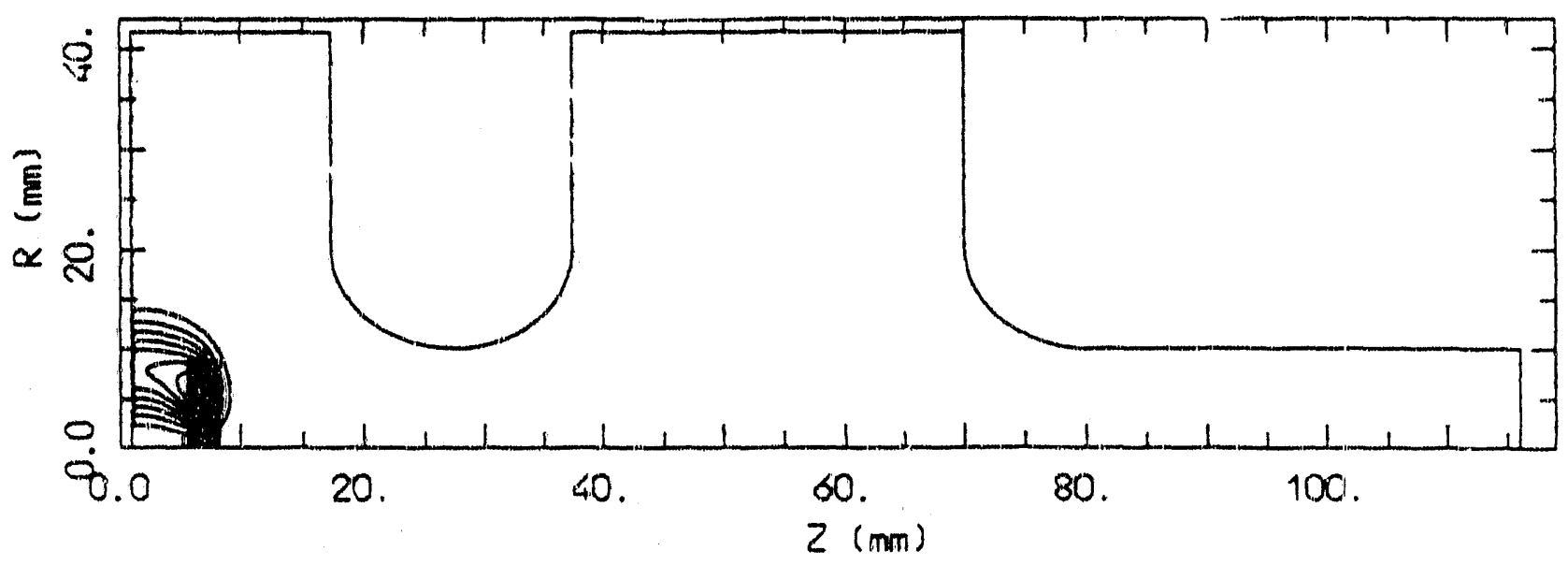

Figure II- 1 shows plot of the $r * \mathrm{H}_{\phi}=$ const lines for $1 \mathrm{nC}$ case, total field. 


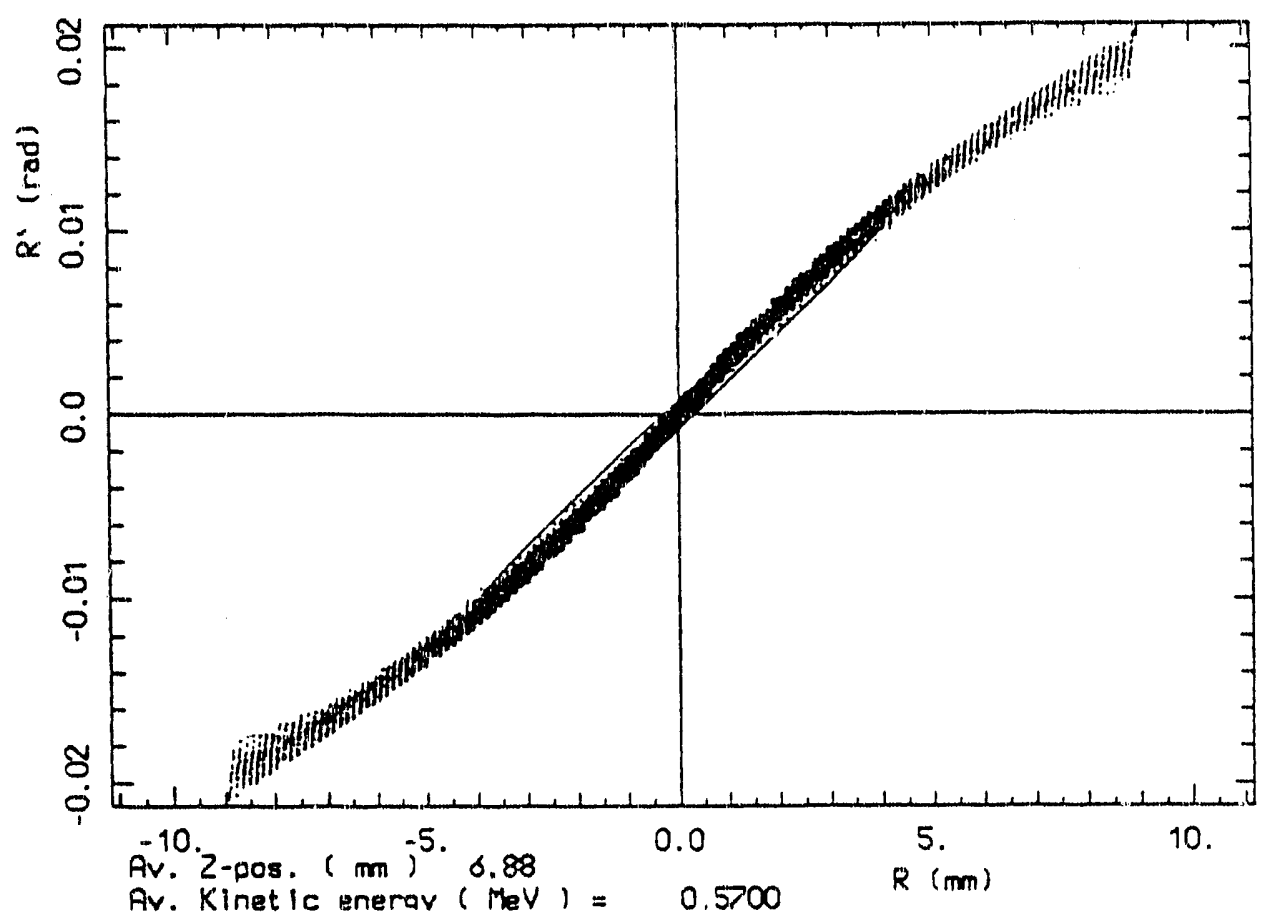

Figure II-2 shows plot of R vs R'; o the transverse phase space. The solid line traces the rms ellipse.

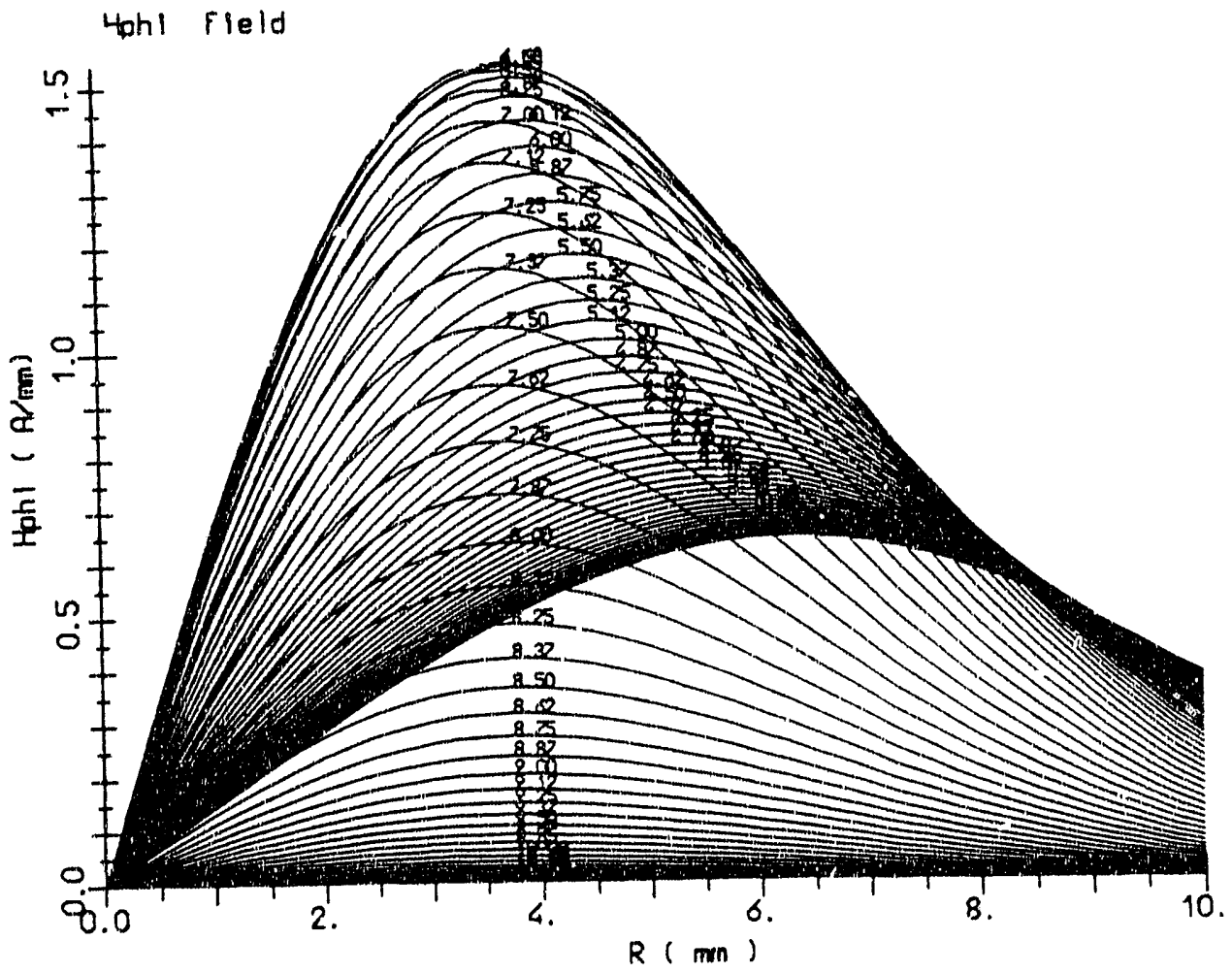

Figure II-3 shows plot of $\mathrm{H}_{\phi}$ vs $\mathrm{R}$, 


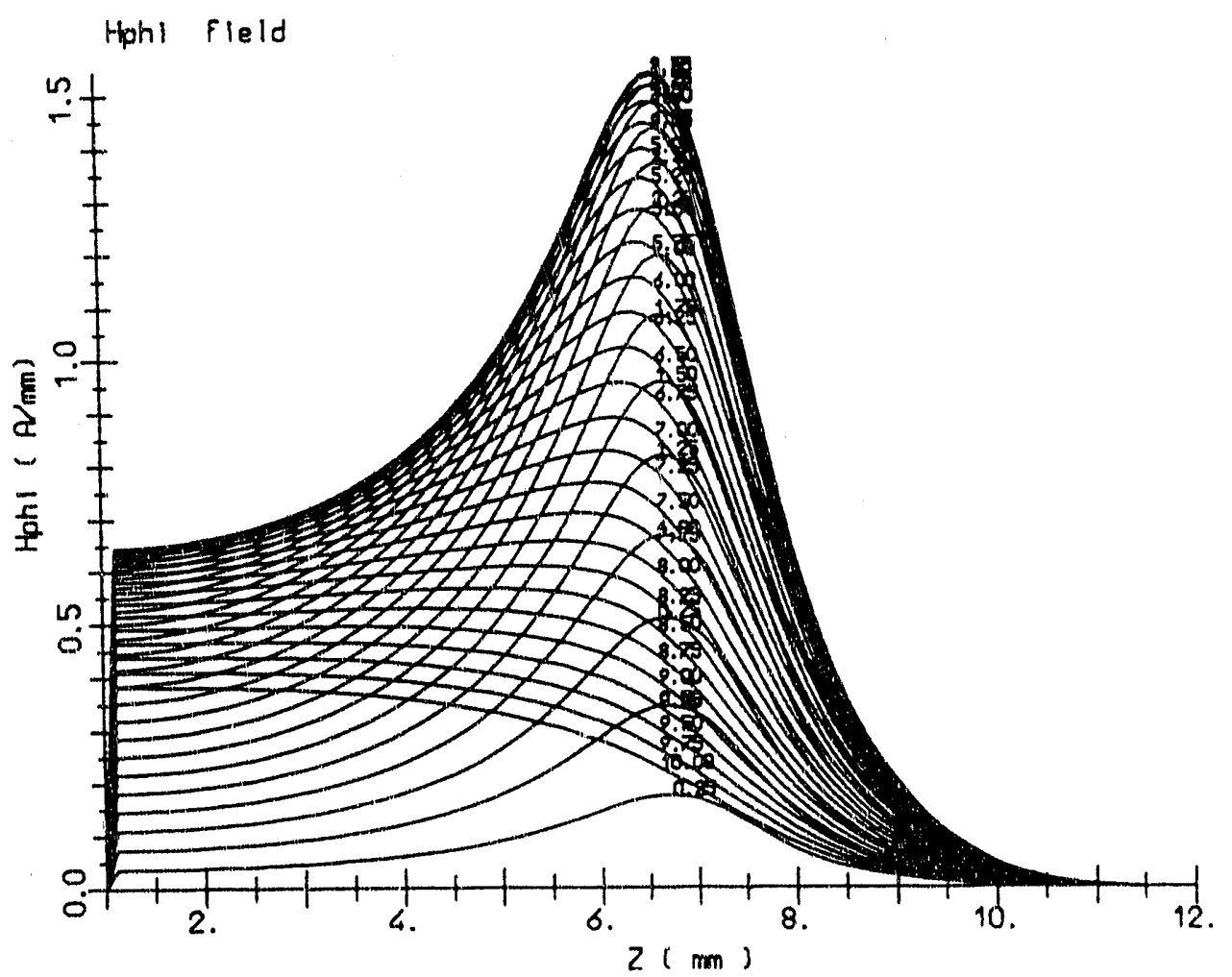

Figure II-5 shows plot of $\mathrm{H}_{\phi}$ vs $\mathrm{z}$,

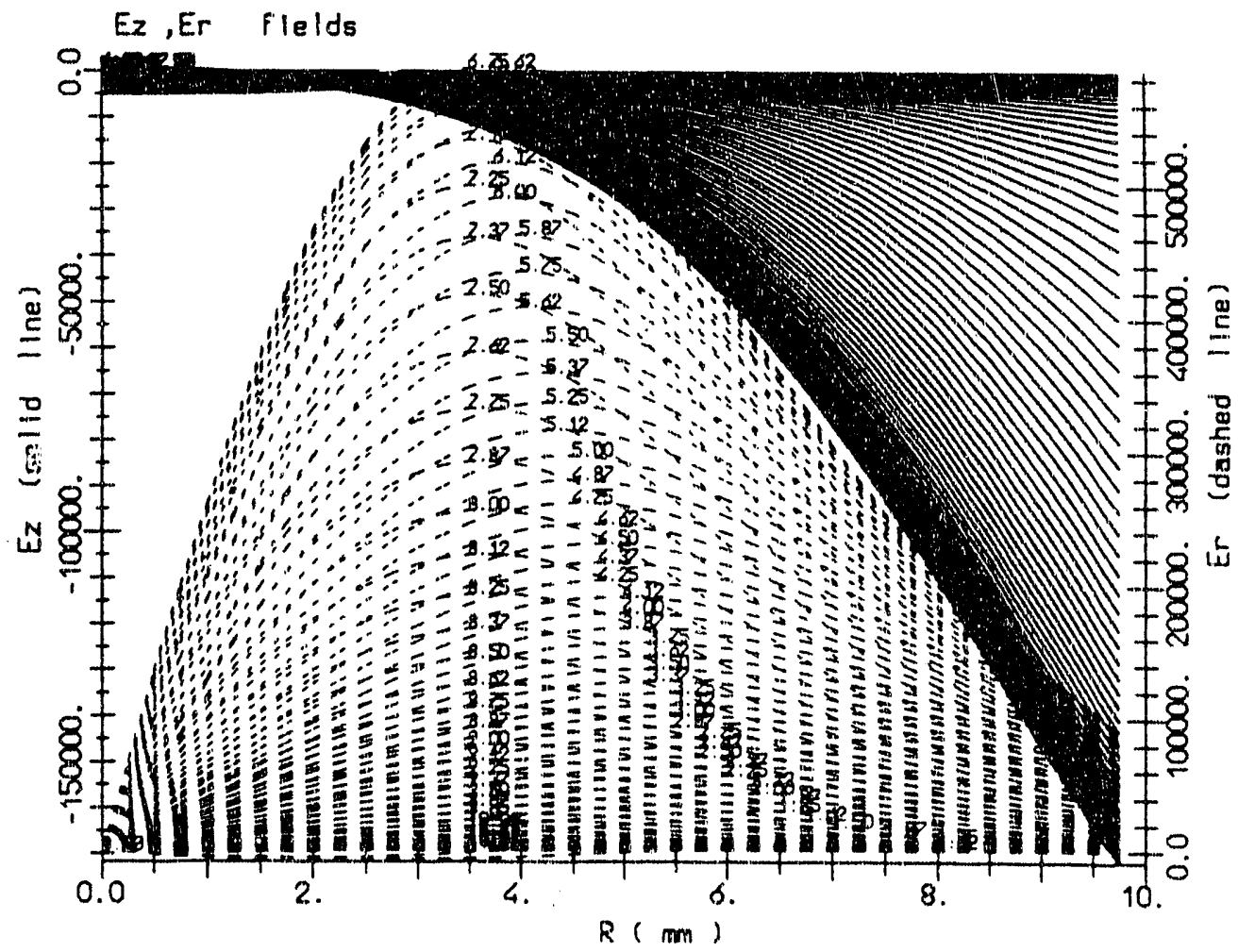

Figure II -5 shows plot of Ez (solid line) and Er (dashed lines) vs r. 


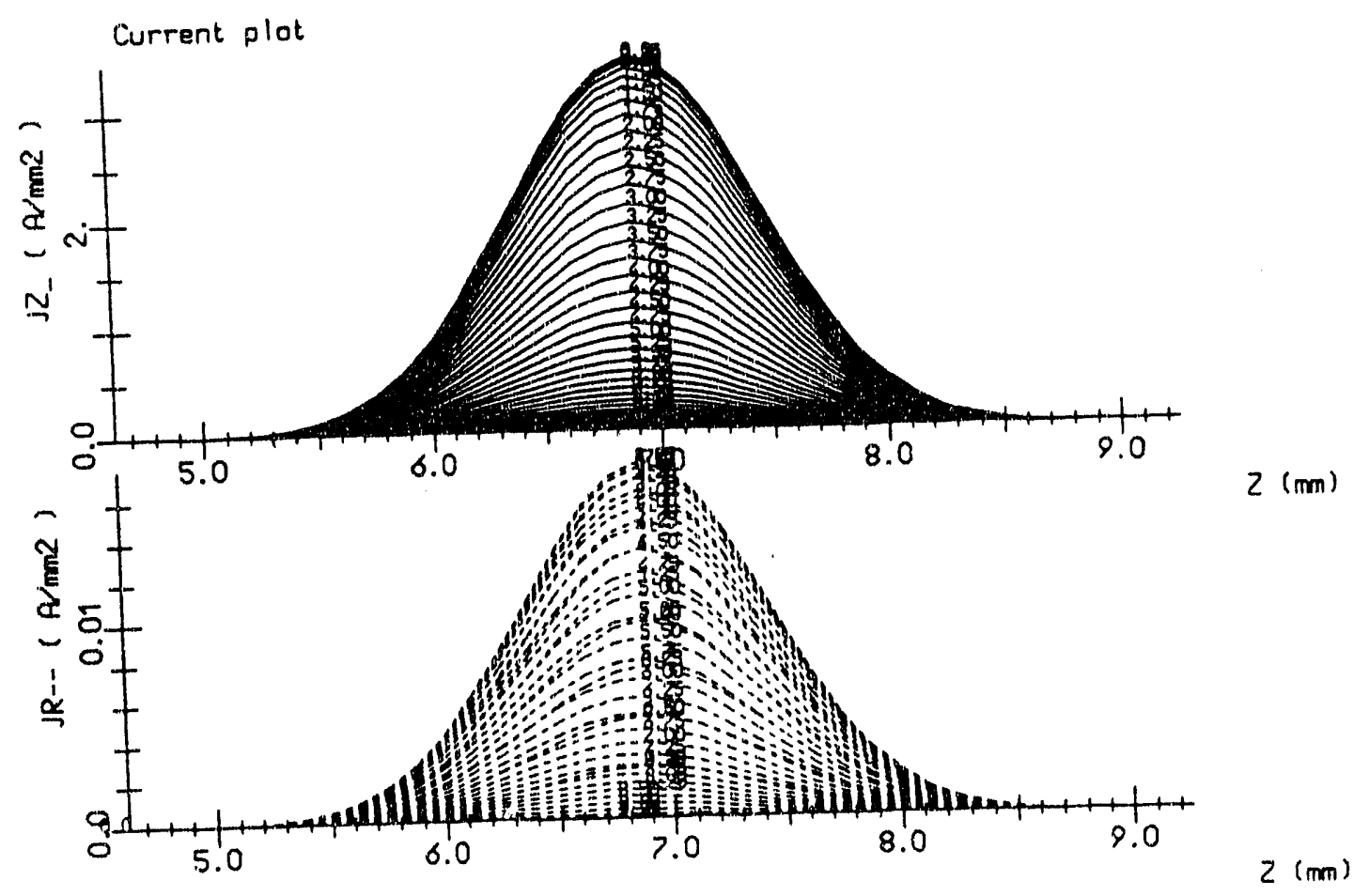

Figure II-6 shows plot of $\mathrm{Jr}$ (solid lines) and $\mathrm{Jz}$ (dashed lines) distributions as functions of $\mathrm{z}$ at different radii.

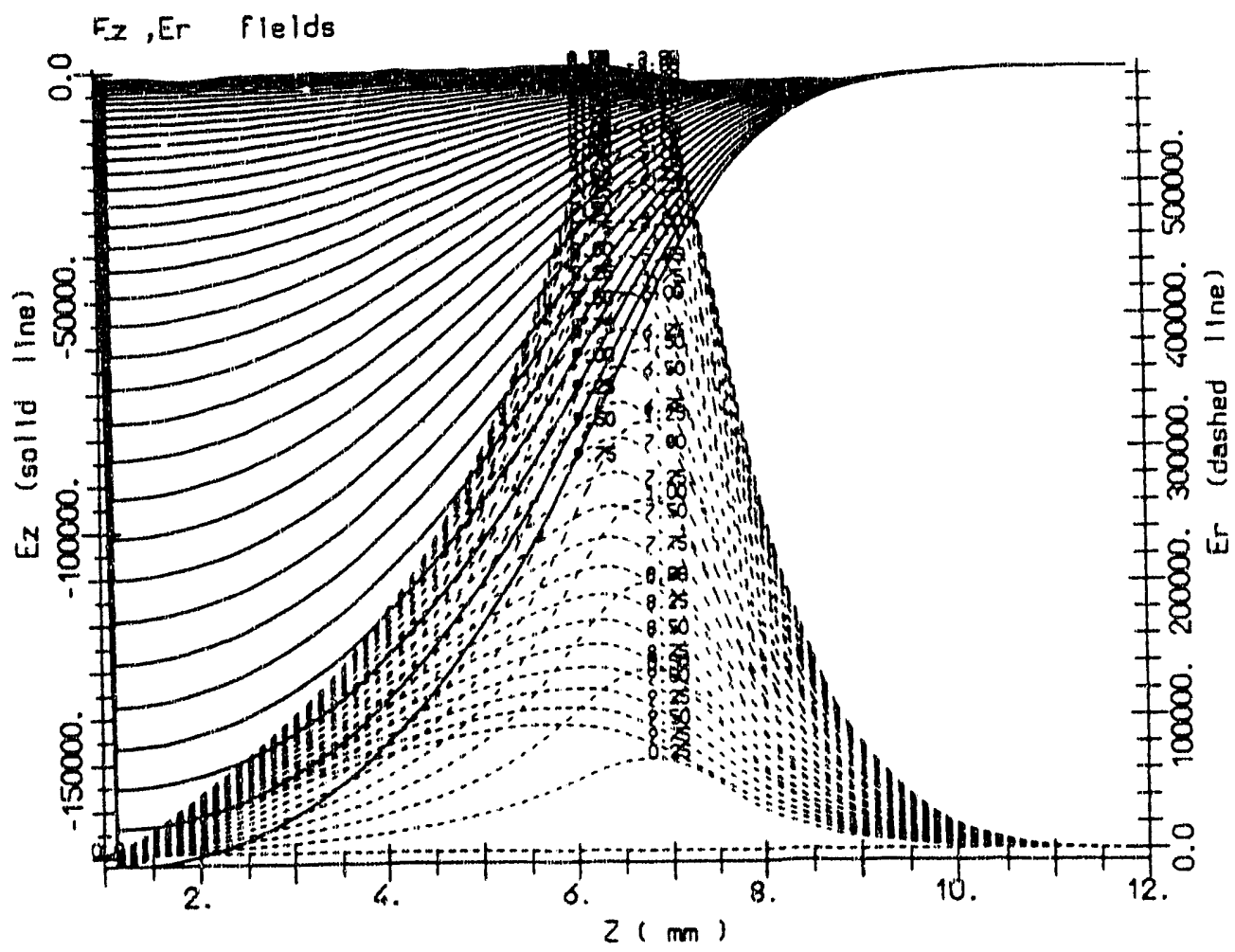

Figure II-7 shows plot of Ez (solid line) and Er (dashed lines) vs Z. 
Figure III-1 to 9, at $\phi_{\text {out }}=123^{\circ}, \phi_{\text {out }}=63^{\circ}$.

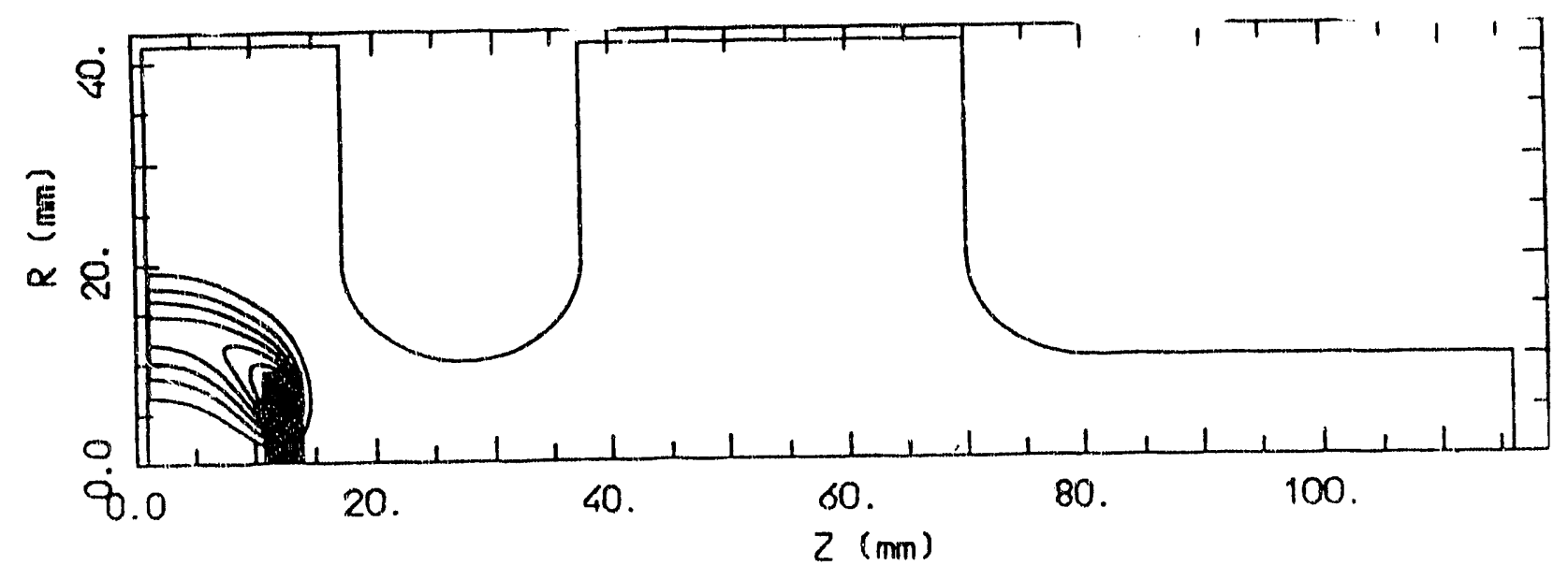

Figure III-1 shows plot of the $\mathrm{r} * \mathrm{H}_{\phi}=$ const lines for $1 \mathrm{nC}$ case, total field. 


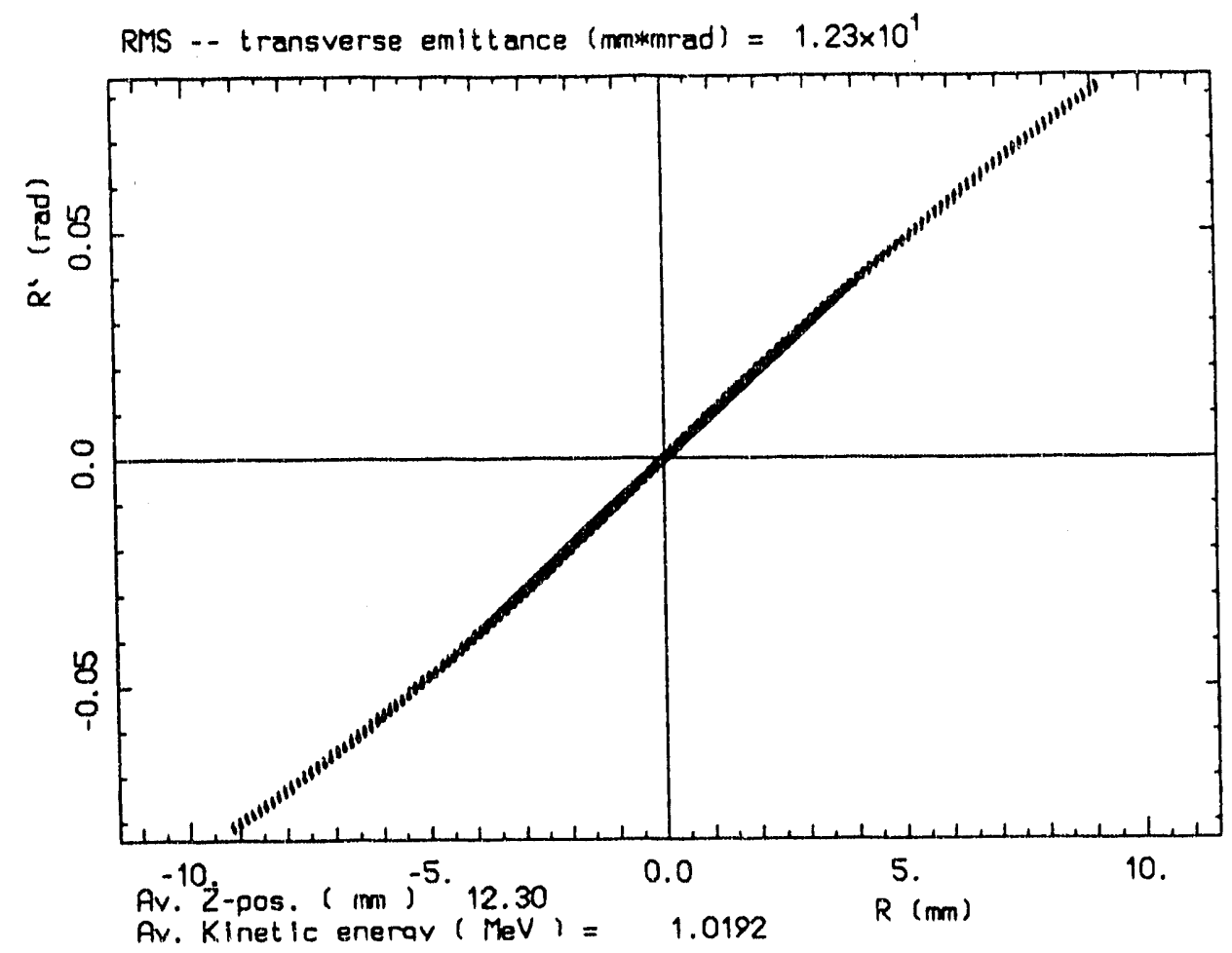

Figure III-2 shows plot of R vs R'; the transverse phase space.

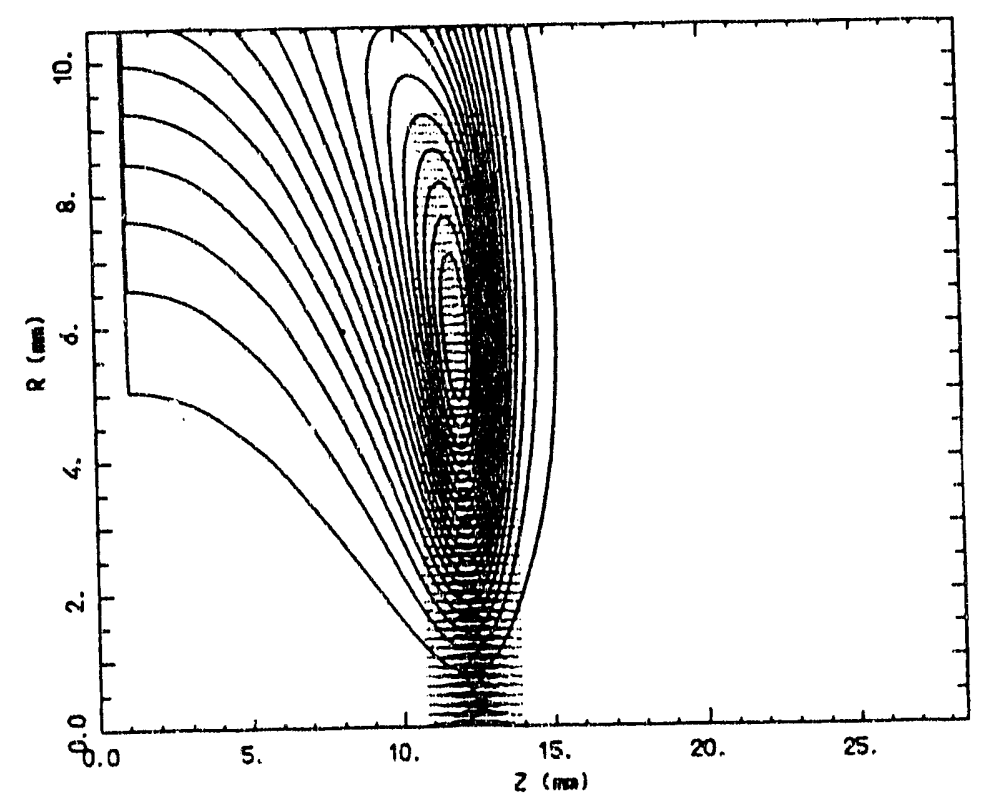

Figure III-3 shows plot of the propagation of the bunch self field produced by the bunch. 


$$
\hat{\Lambda}
$$




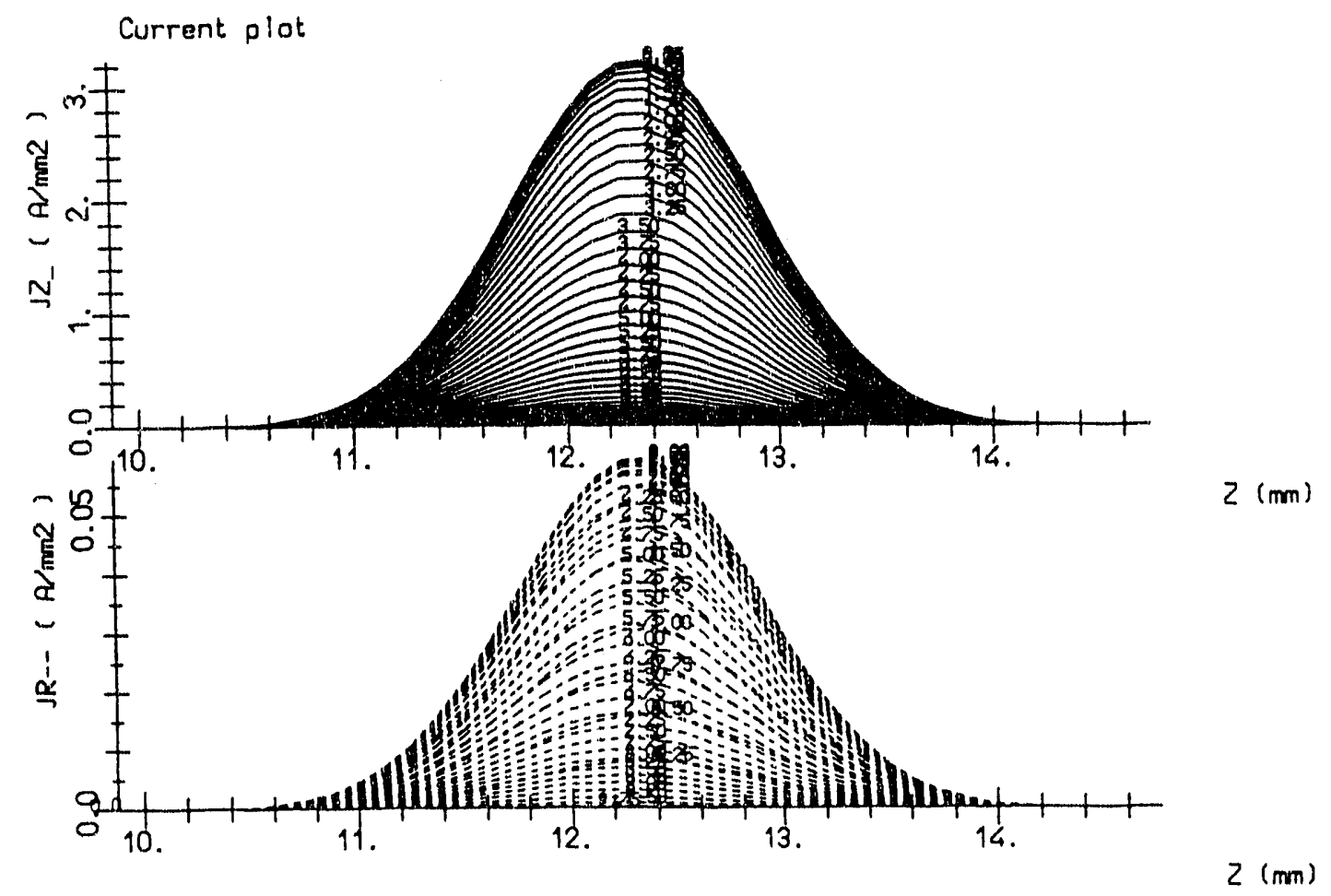

Figure III- 6 shows plot of $\mathrm{Jr}$ (solid lines) and $\mathrm{JZ}$ (dashed lines) distributions as functions of $\mathrm{z}$ at different radii.

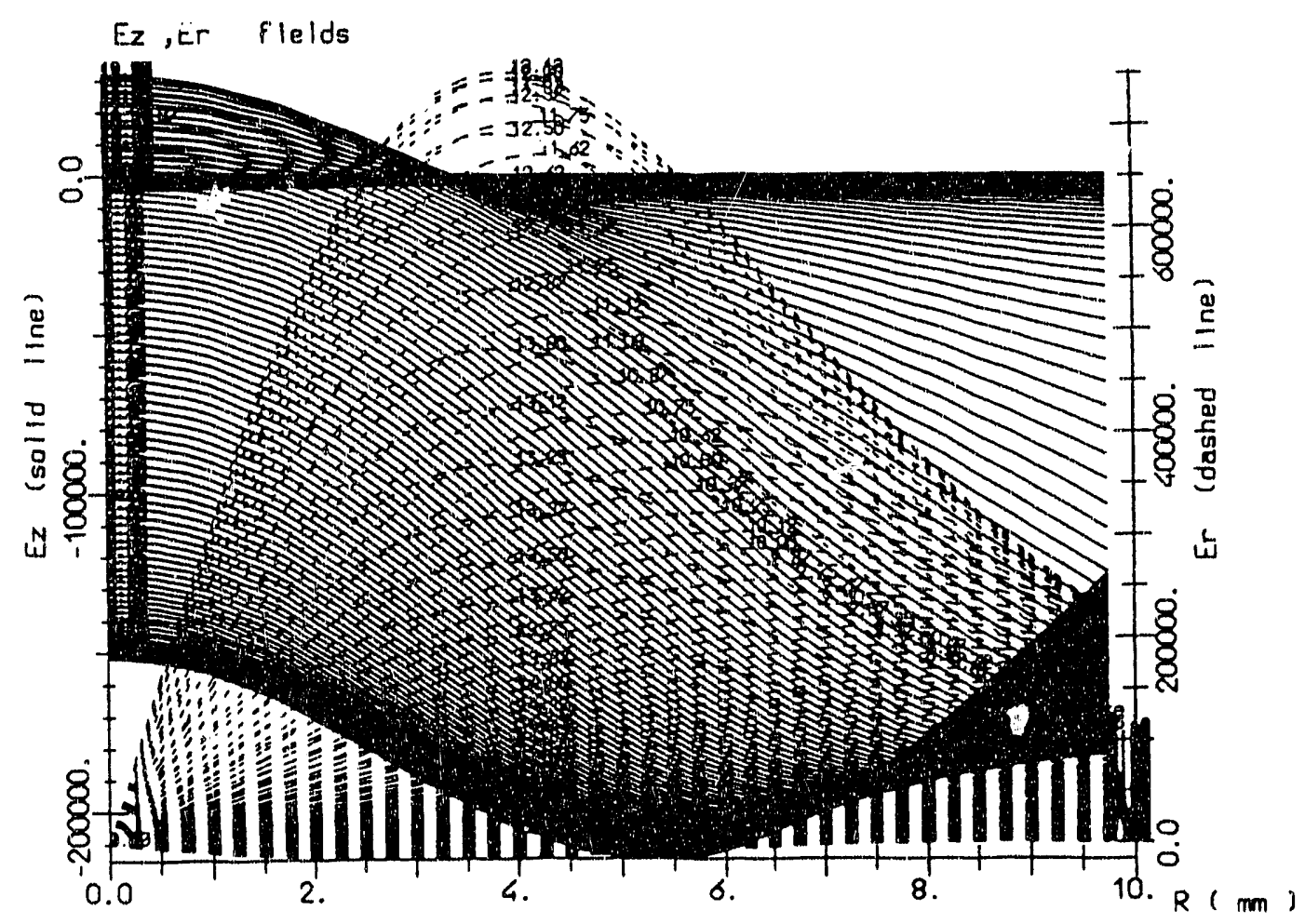

Figure III-7 shows plot of Ez (solid line) and Er (dashed lines) vs r. 


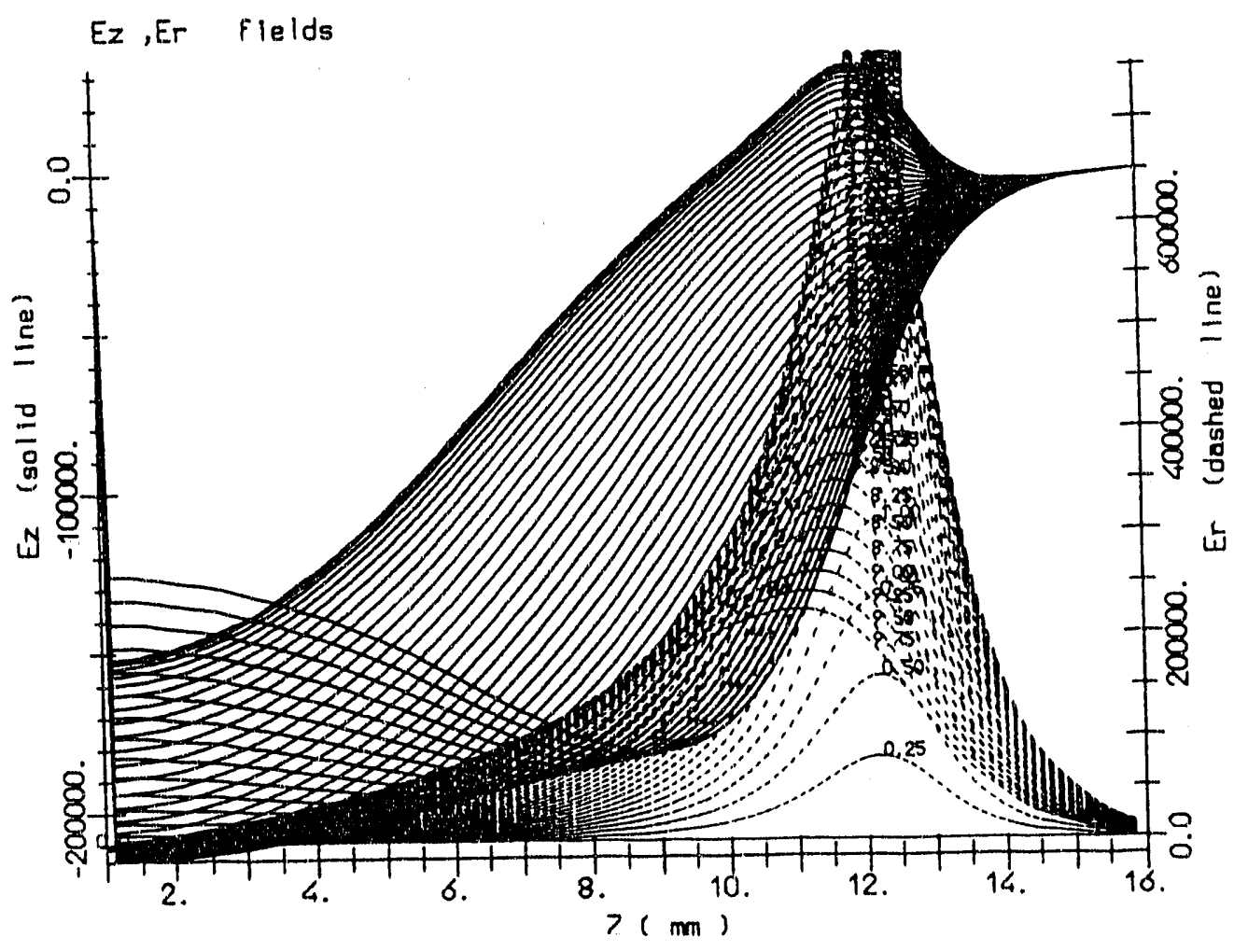

Figure III-8 shows plot of Ez (solid line) and Er (dashed lines) vs Z.

Charge density - dive plot

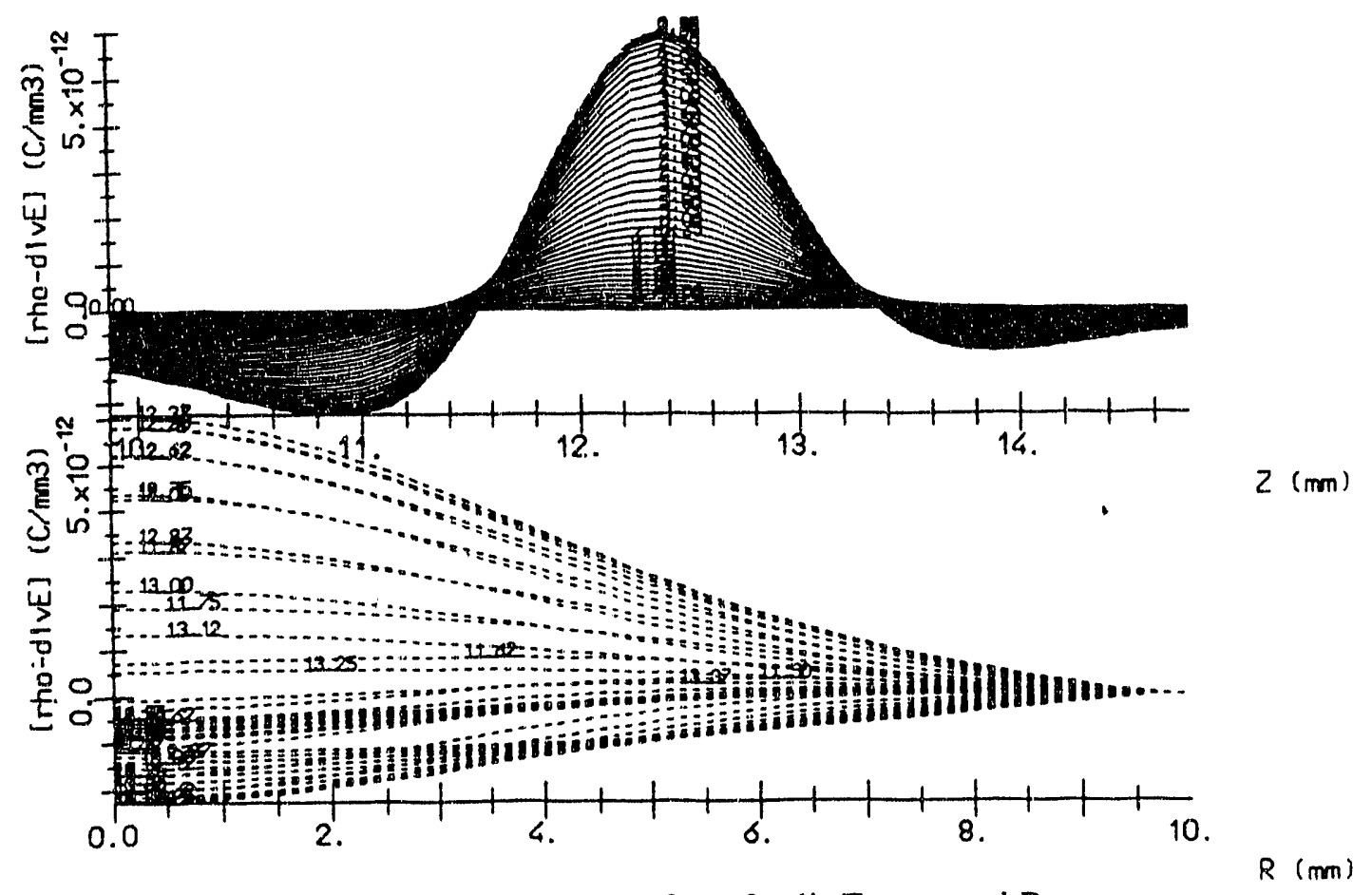

Figure III-9 shows plot of pdivE vs $z$ and $R$. 


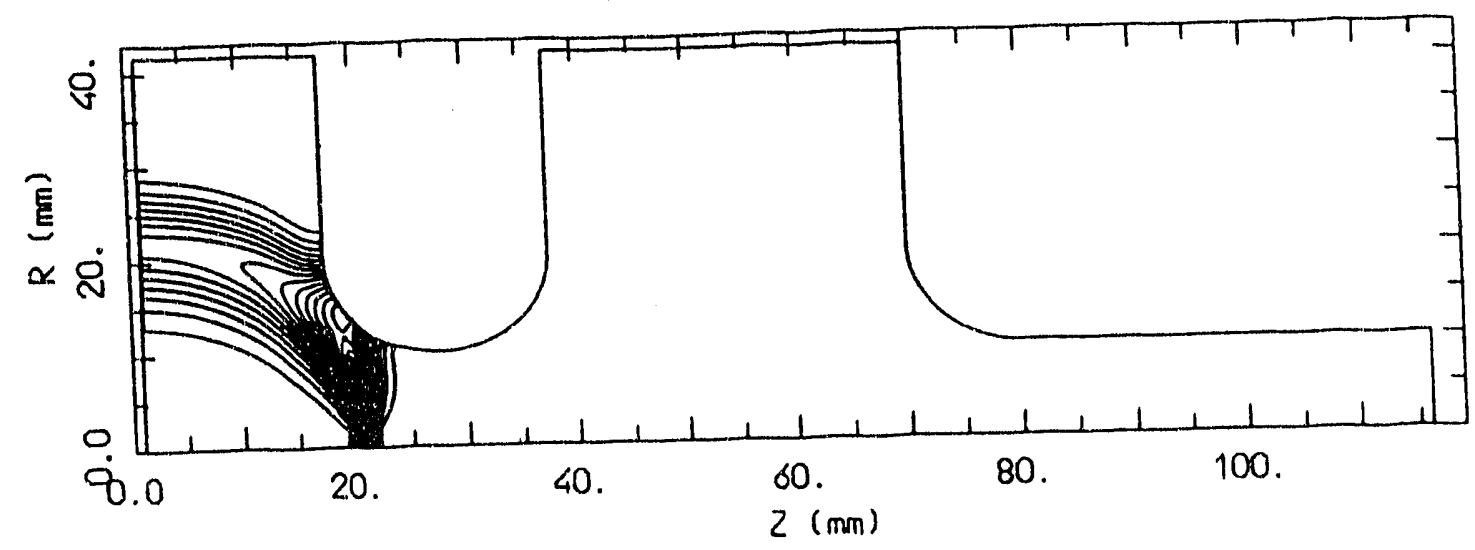

Figure IV-1 shows plot of the magnetic component of the bunch self - field.

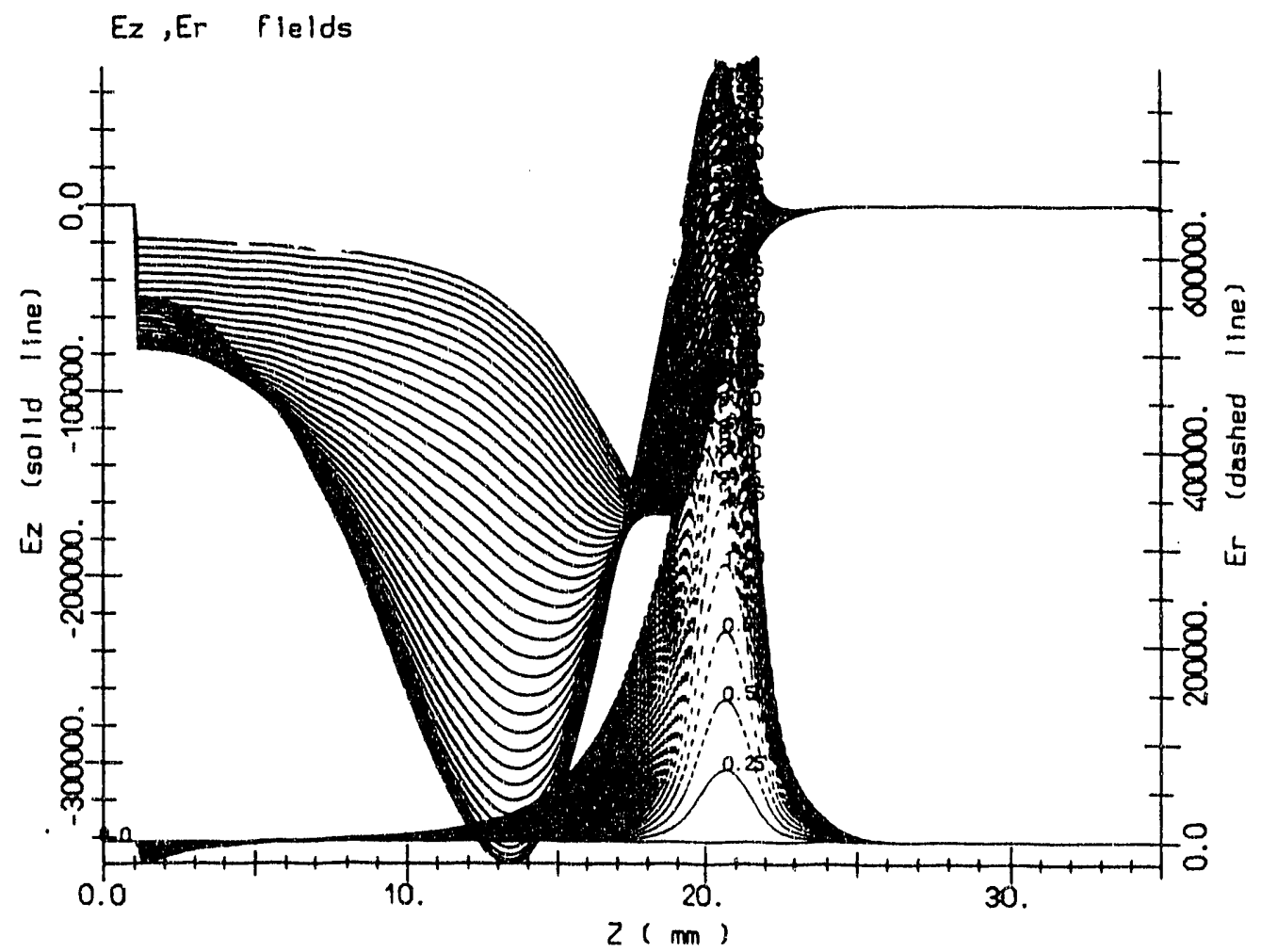

Figure IV-2 shows plot of Ez (solid line) and Er (dashed lines) vs $z$, the electric component of the bunch self field, at $\phi_{\text {out }}=152.905^{\circ}$. 
Figure V-1. to 8, at $\phi_{\text {out }}=243.039^{\circ}, \phi_{\text {in }}-63^{\circ}$.

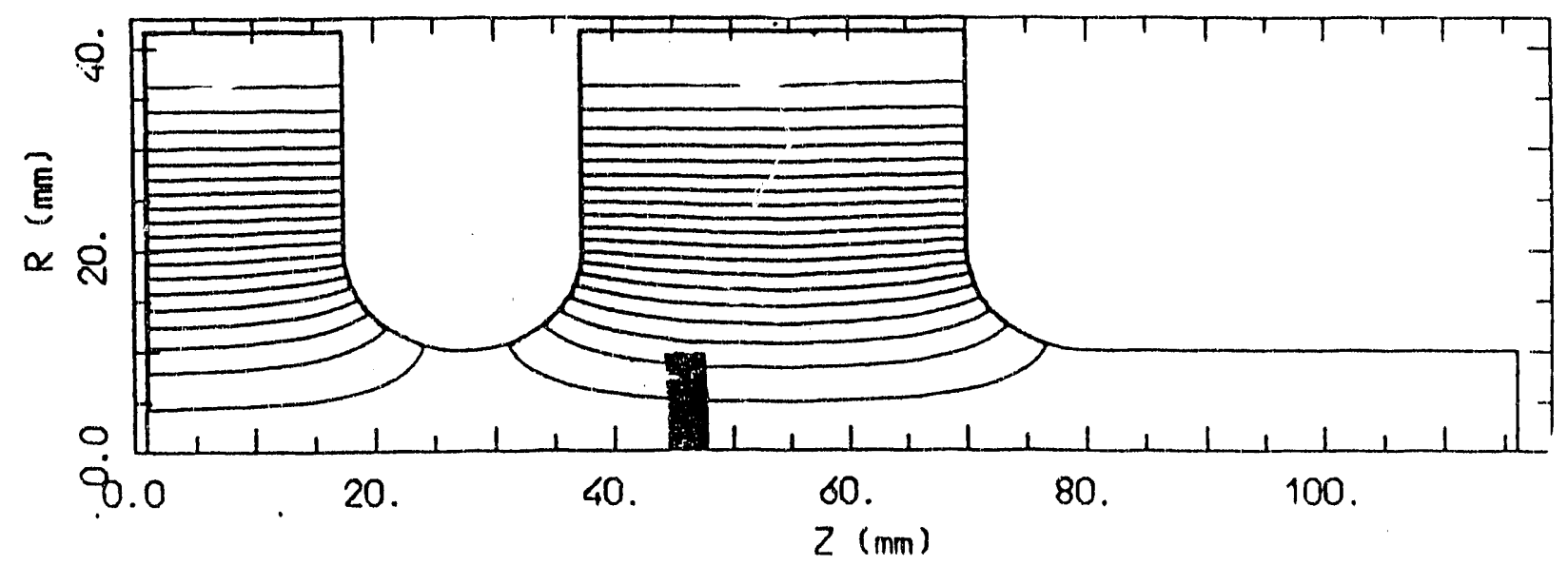

Figure V-1 shows plot of the $\mathrm{r} * \mathrm{H}_{\phi}=$ const lines for $1 \mathrm{nC}$ case, total field. 


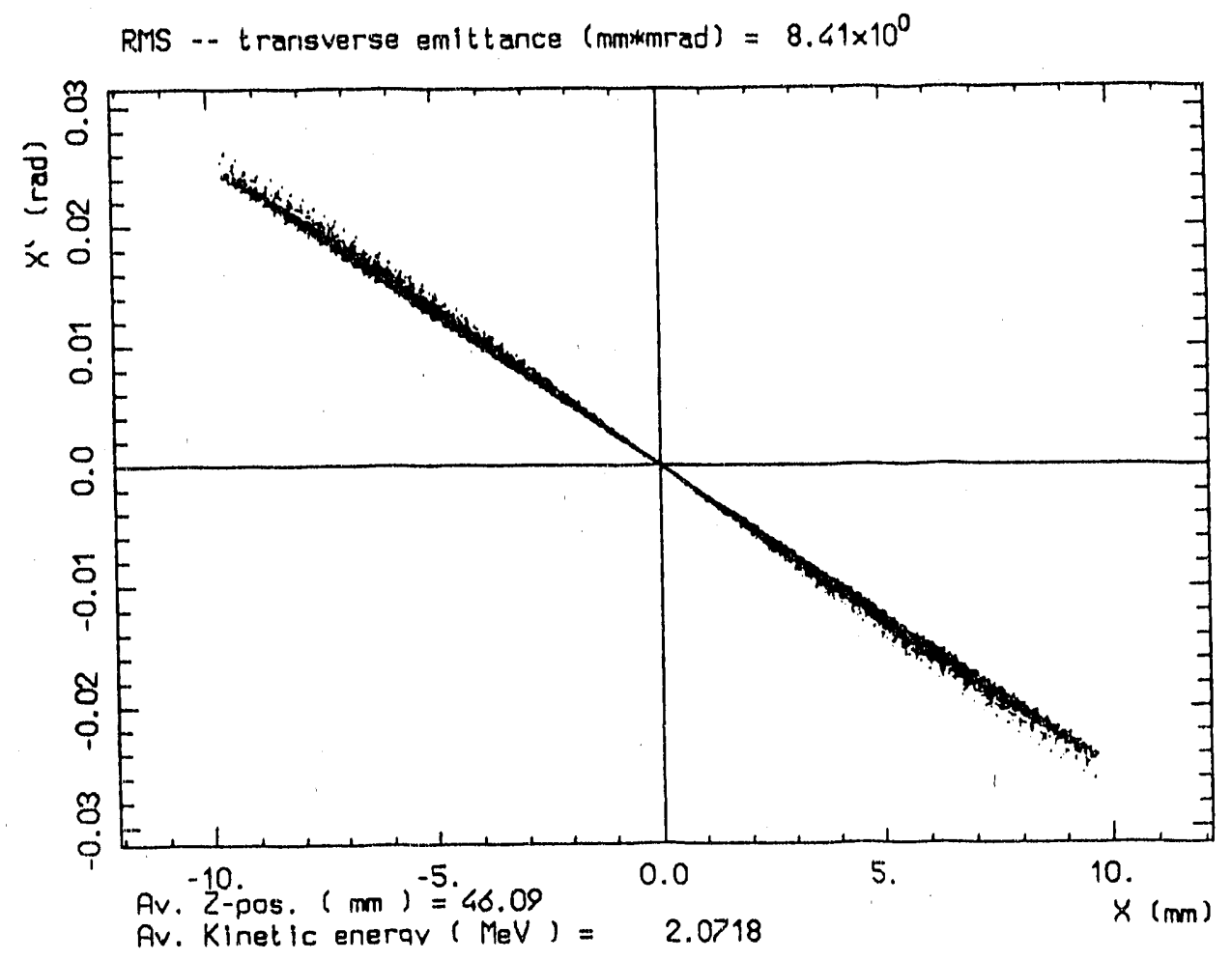

Figure V-2 shows plot of $\mathrm{X}$ vs $\mathrm{X}^{\prime}$; the transverse phase space.

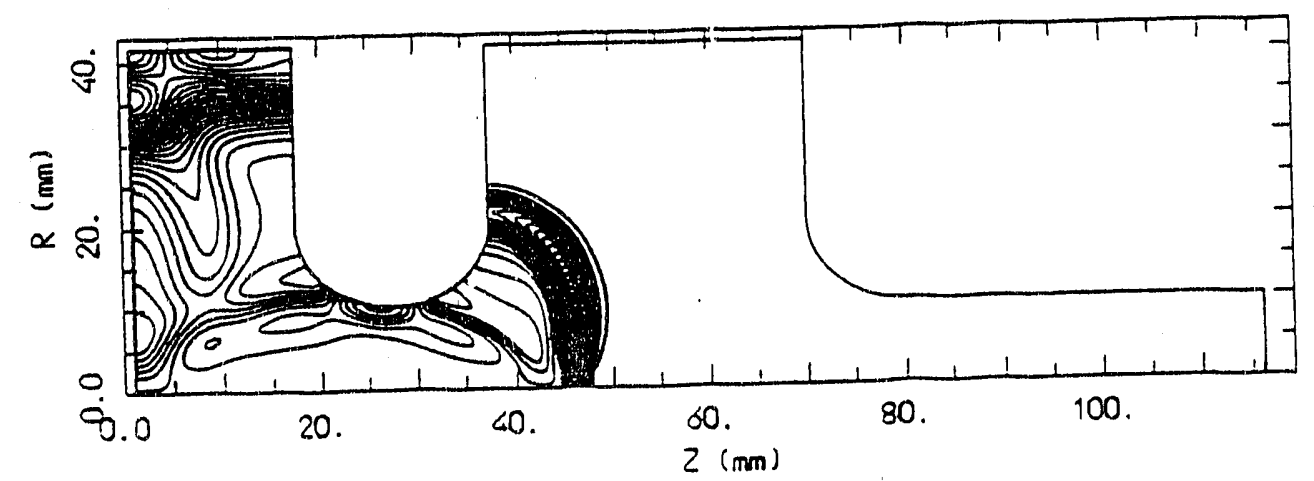

Figure V-3 shows plot of propagation of the bunch self field produced by the bunch. 


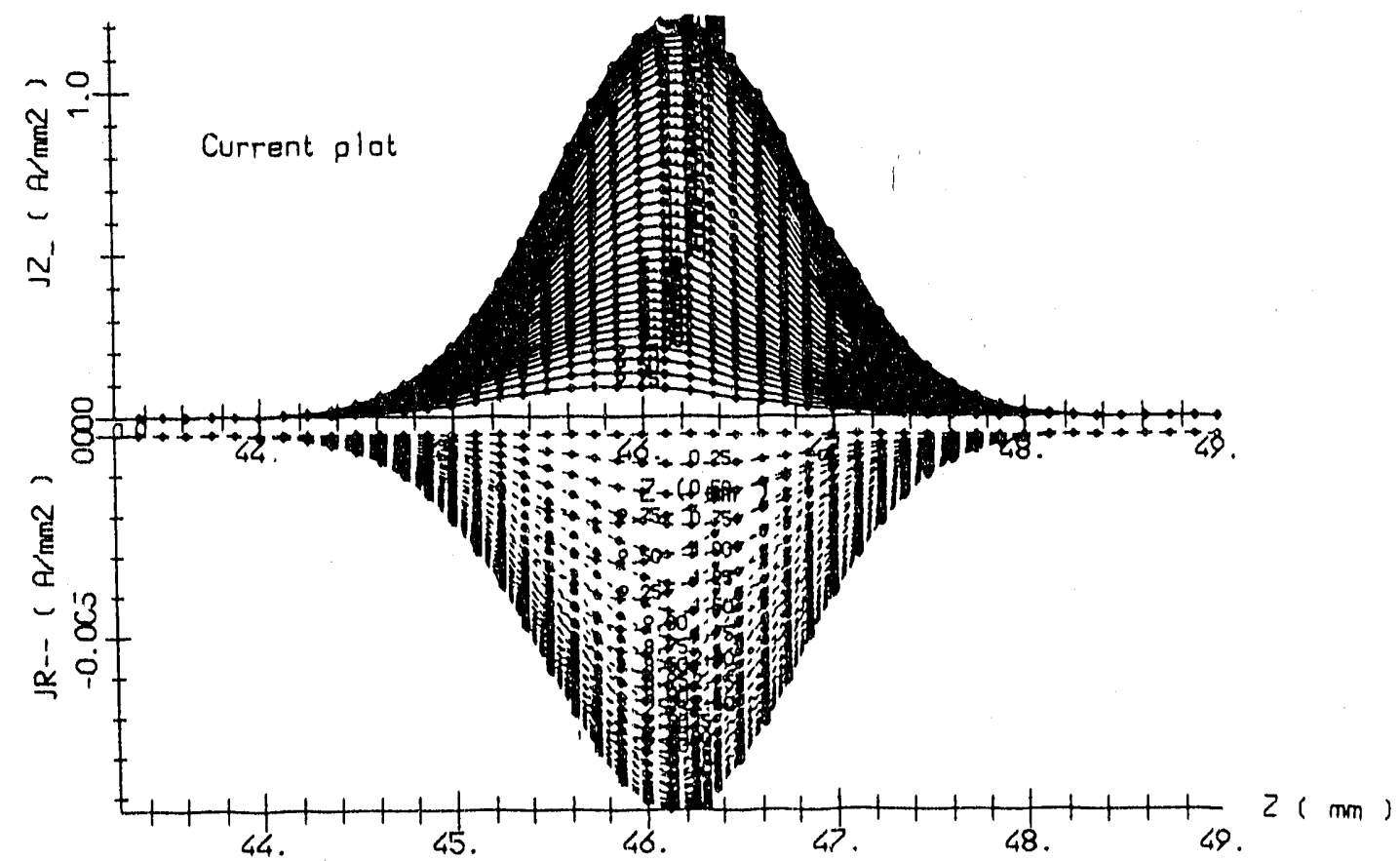

Figure V-4 shows plot of $\mathrm{Jr}$ (solid lines) and $\mathrm{Jz}$ (dashed lines) distributions as functions of $\mathrm{z}$ at

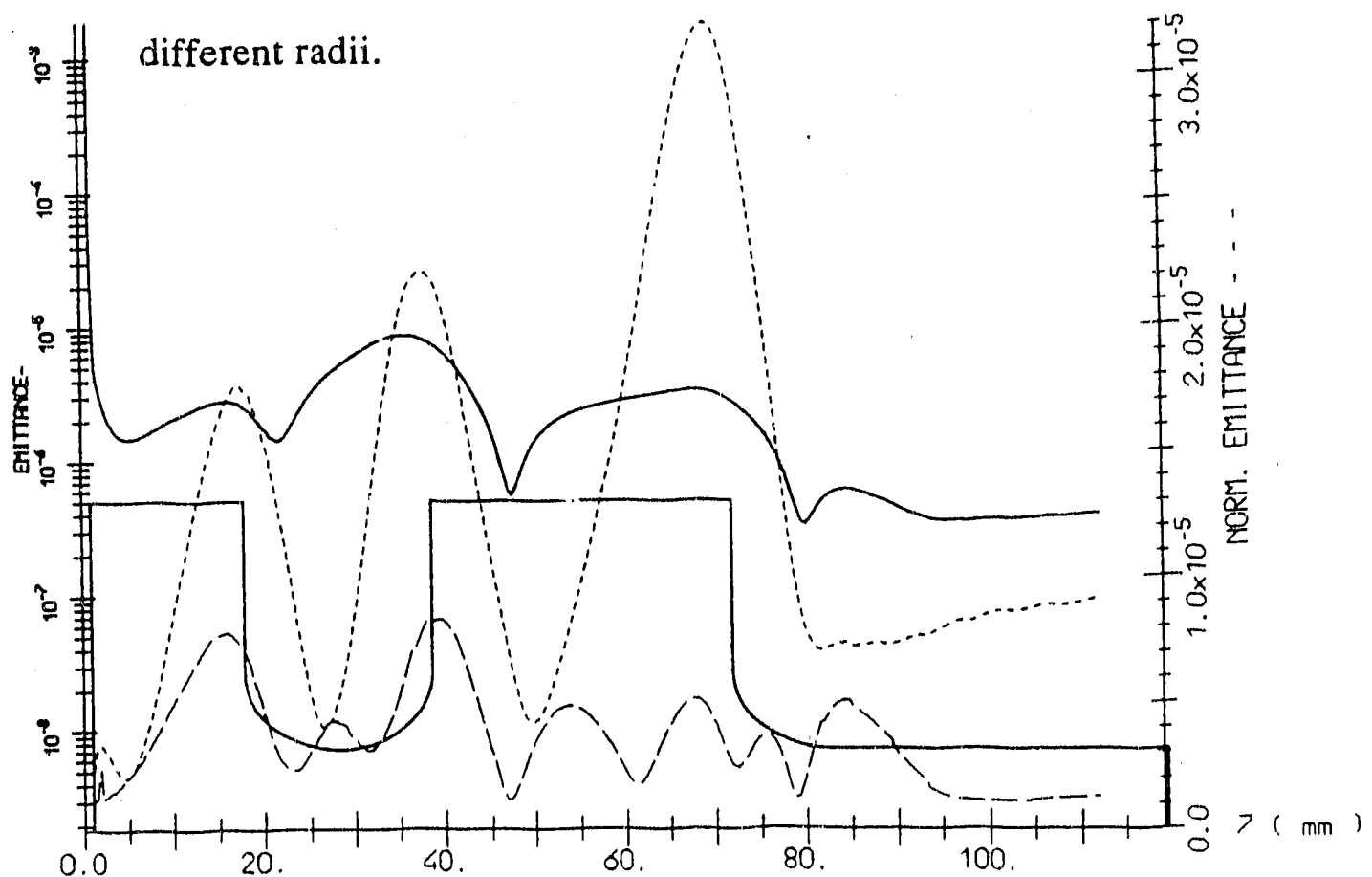

Figure V-5 shows plot of Transverse Emittance vs z. Dashed line shows the normalized emittances $\sqrt{\left(x^{2}\right\rangle\left\langle p_{x}^{2}\right\rangle-\left\langle x p_{x}\right\rangle^{2}}=\varepsilon_{n} ;$ Solid curve line shows the emittance $\varepsilon=$ $\sqrt{\left\langle x^{2}\right\rangle\left\langle x_{1}^{2}\right\rangle-\left\langle x x^{\prime}\right\rangle^{2}}$ and third curve $\left(\varepsilon_{n}^{c e n t r a l}\right)$, gives $\varepsilon_{n}$ associated to the central slice of the bunch. 


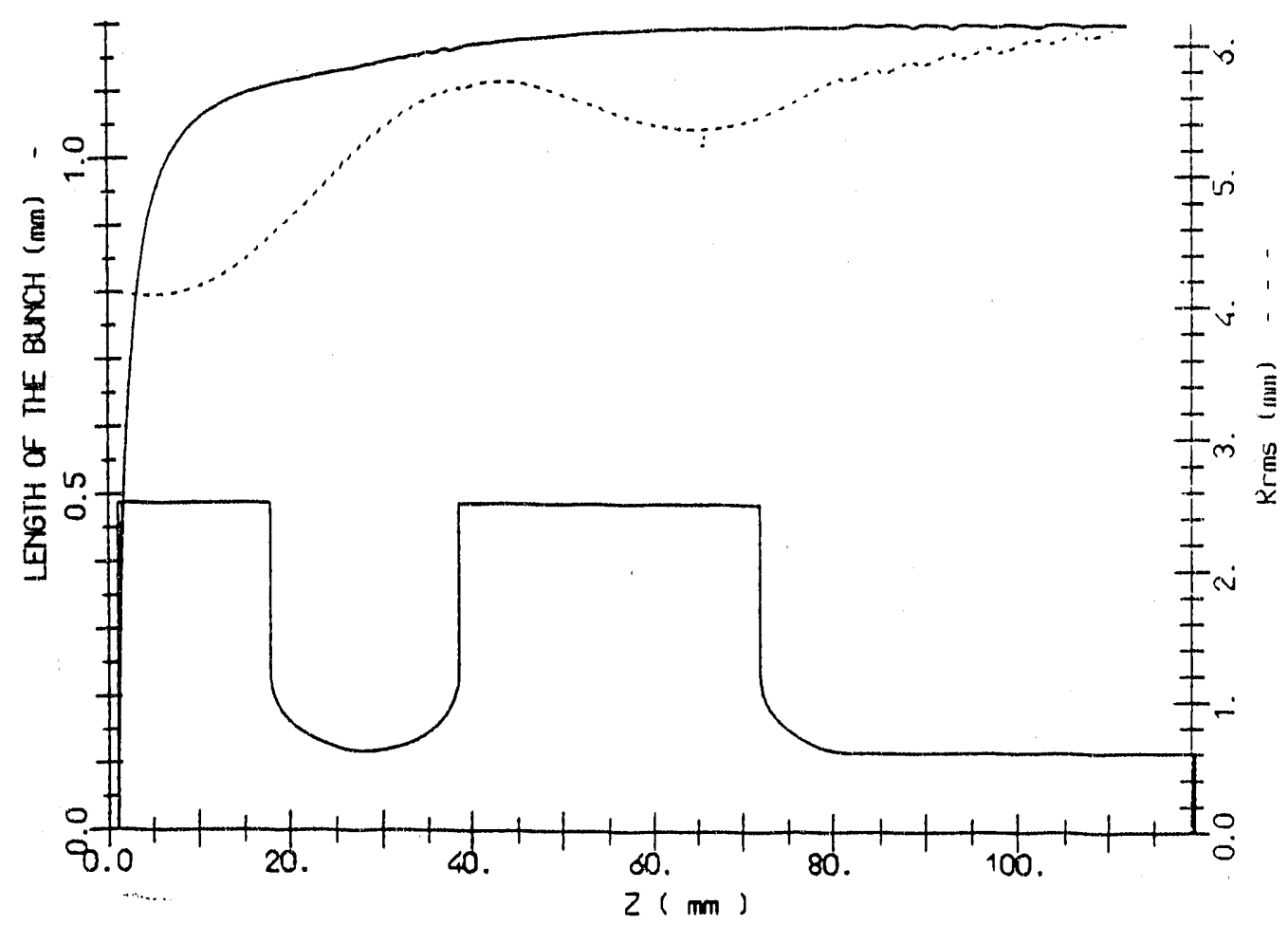

Figure V-6 shows plot of length of the bunch and Rrms vs $z$.

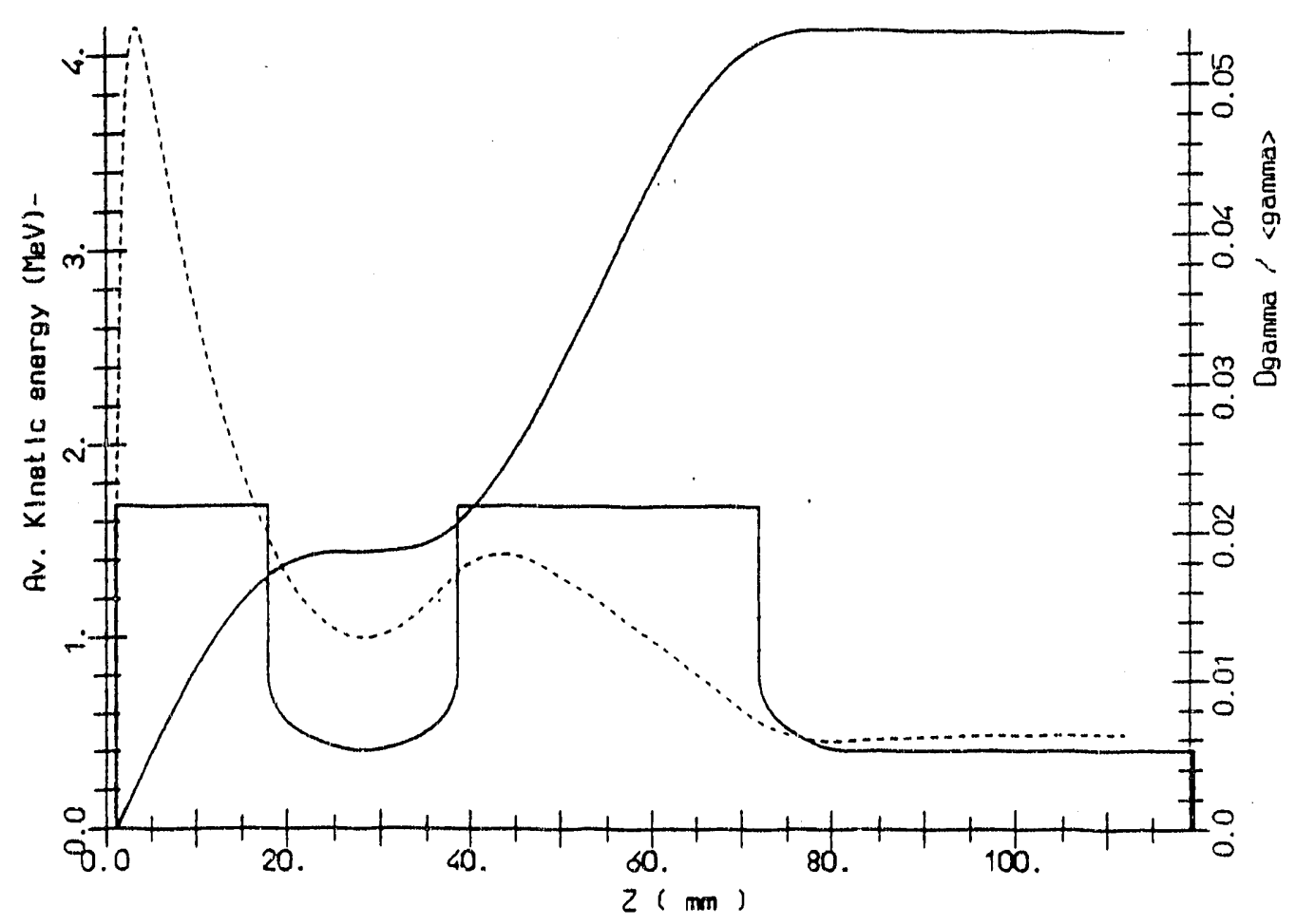

Figure V-7 shows plot of Average Kinetic Energy (solid line) and $\Delta \gamma /\langle\gamma\rangle$ (dashed line), vs $z$. 
Av. Current $(A)=1,48.39$

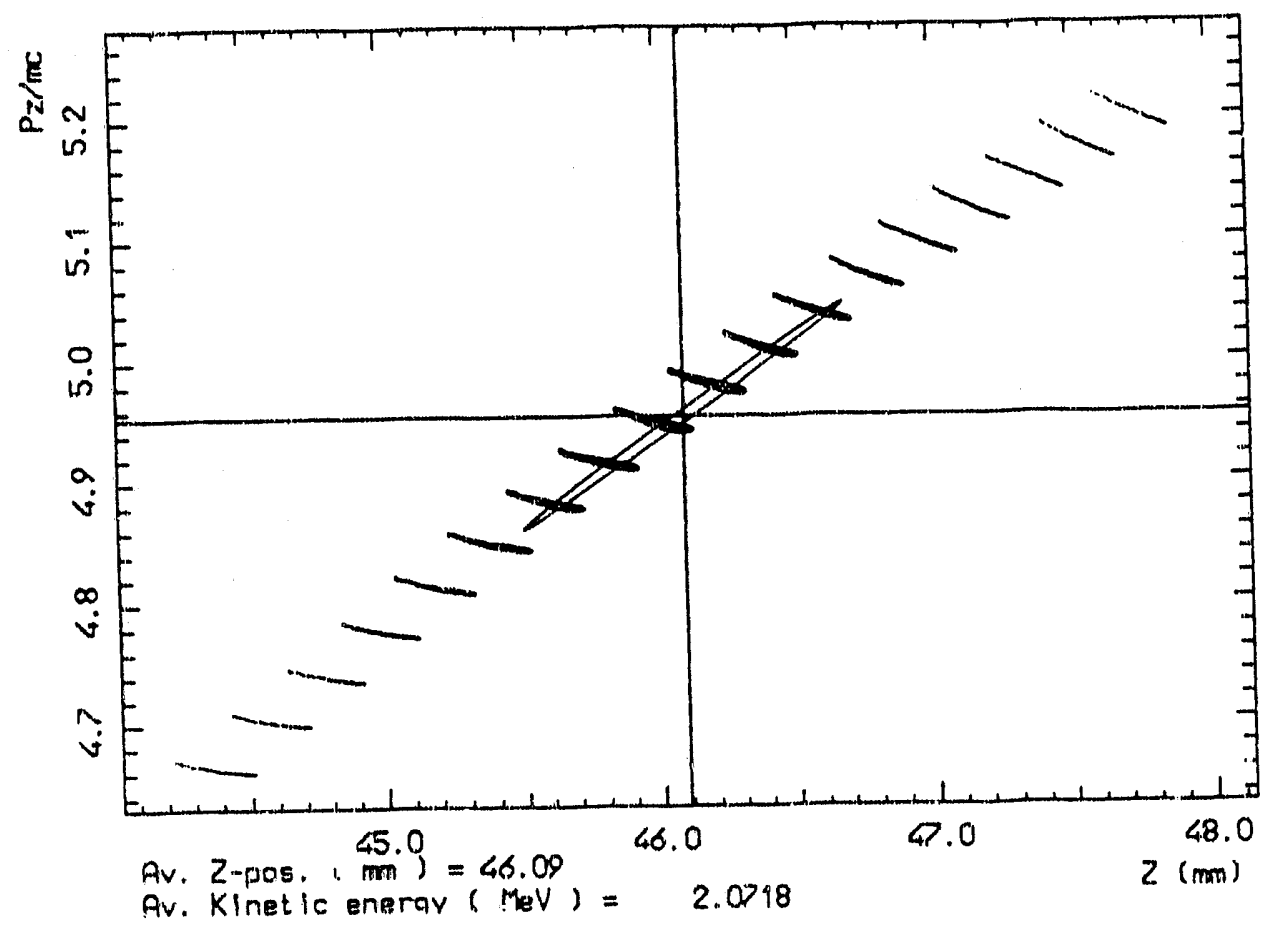

Figure V-8 shows plot of $\mathrm{Pz} / \mathrm{mc}$ vs $\mathrm{z}$. The solid line is the rms ellipse. 


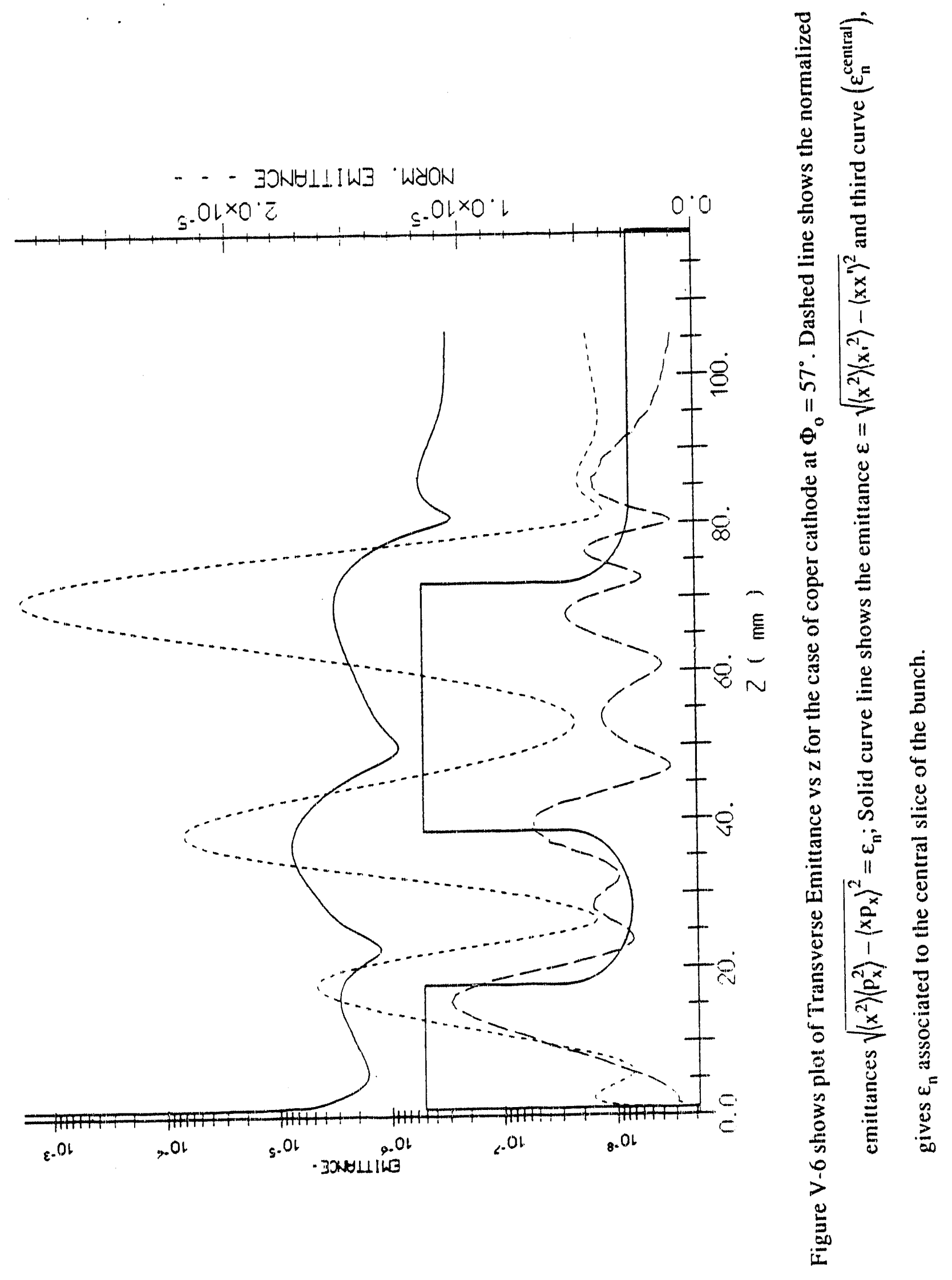



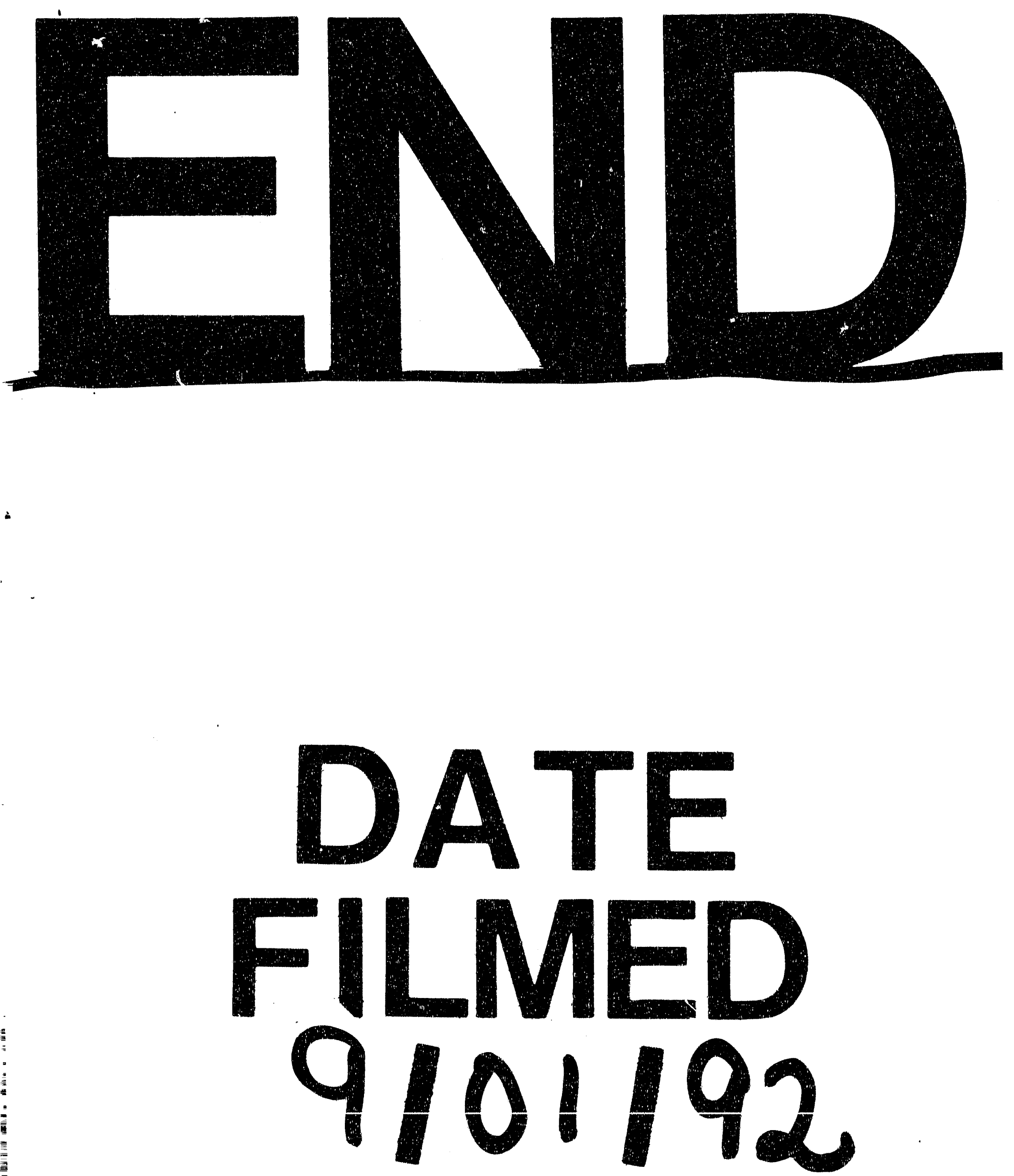


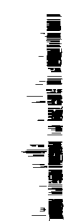

